\title{
TLEN POLIKEH KIETICHIWAH OMPUALLI WAN EYI MOMACHTIYANIMEH TLEN AYOTZINAPA: ¿SE TLAMANTLI TLEN TLAIXNEXTILISTLI IKA TLAYEKANALIH TLEN KINEKIH IPAN TEPOSTLAPOLIMEH TLAKUALTLALISMEH TLEN TLANAWATIHETL?
}

\author{
(Tlakuilolli tlen se moihilistlih)
}

Miguel ONTIVEROS ALONSO*

\section{Tlen mochihki}

Ipan sempualli wan chikuase tlen septiembre tlen ome xipilli wan matlaktli wan nawi, tonatih tlen mochihki, eyi momachtianimeh tlen ne kaltlamaxtiloyah Normal Rural Raúl tlen Ayotzinapa, ipan ne Mexkotlalli tlen Guerrero, kinikixtilke inih yoloh, sempoalli wan makuilli elkeh tlakohkolmeh wan ompualli wan eyi polike'. Wan kitzintemohke ipan ne Fiscalia Gerneral tlen altepetl Guerrero wan tlen ne- Procuraduría General tlen ne República asitokeh ipan tetzakuilli tlen nakpualli wan matlaktli wan chiknawi towampoyomeh, ika iniwantih eltok tlen ne eltoyah weyihtekichihetl tlen chinankomeh tlen Iguala wan isiwah, kehnopah kampa kenikatzah miyakeh tlen tekichihemeh tlen ne tekiwatlanawatihetl tlen ne tlatilantokeh tlen Iguala wan Cocula. Nohkia eltok nemilistli tlen miak tlakayomeh tlen -kehnopah motokaxtihtokeh- tekilistli

\footnotetext{
* Ni tlakuilolli elih tlen ne tlahtolixnextilli tlen monextihih, ne nawi tlen febrero tlen 2015, ipan ne Centro tlen Estudios tlen Derecho Penal wan Procesal Penal Latinoamericano (CEDPAL) tlen ne Georg-August-Universität Göttingen, Alemania, tlayekanketl ika ne Prof.Dr.Dr h.c. Kai Ambos. Se axtowih tlahtolkuapilih, achih tlachinehotzih, moixnextis ipan «Revista Penal Centroamérica».

* Becario tlen Fundación Alexander von Humboldt ipan ne Instituto tlen Derecho Penal tlen ne Universidad tlen Bonn, ika ne weyihtekichihetl weyihkaltlamaxtiloyah tlen Dr. Dres. h.c. Urs Kindhäuser. Tlahtolitlahtlanketl (SNI) ipan ne Instituto tlen Investigaciones Jurídicas tlen ne Universidad Autónoma tlen Chiapas, México.

${ }^{1}$ Ne Procuraduría General tlen ne República kiitohtok tlen ne tilih wan omitl kiasitok iwikal, ika kentzih, tlen se tlen ne normalistas politokeh. Echkah tlen ne kintlatike tlen tlaihyowiyah tlasentilimeh tlaxelolimeh -kenihatzah onkah nikanih ika ne momaxtianimeh wan tlen kistokehyah tlen ne Normal Isidro Burgos tlen Ayotzinapa- wan tlen ne tlawel tlanextilli tlen kipiah ipan ne weyihtlayenkalsahetl; Schroeder, Friedrich, Christian; Zweifel an der Asozialenkriminalisierung in der DDR; JZ; 1987; p 1004: se tlen kiixnextiyah, wan axkualli tlaixnextilistli tlen sakuamelawakayotl tlen ne DDR, eltok ne tlaiyokatekohkolihetl tlen ne iniwamotlaltoh. Nohiah onkah ipan ne Unión Soviética ika ne tekitl mochitok ika iniwantih tlen ne -kehnopah kintokaxtihtokeh«parásitos». Ne sansehotlalistli (Asozialität) moitayahyah -ika iwikal tlen ne temiktihetl - ika se kenihatzah moitowah achih miak tlen iyolohtlaixnextilli nochimeh temahmahtih.
} 
tlen ne «guerreros unidos» $»^{2}$. Ika itlamilis iwaya ne tlaixnextilistlih tlen yainih tlamantlih wan mokalakis ipan se kuanehnemilistli, ninekih kixnextis ne tlen tlaixnexamostlahtolli3:

1. Sekih kinitzkitokeh -namah kitlatelwiliheh- kiitohtokeh tlen «procedieron» kichiwaseh ne tekichihetl ika se tlaixnexkayotilistli xitlawak tlen tlatilanketl tlen chinankomeh, tlen ahiah tlanawatitoyah mamochiwah ika ne normalistas tlen Ayotzinapa tlen mamoasikah ipan miyaken teposnehnenkemeh ipan ne tlatilankok tlen Iguala.

2. Ne Procuraduría General tlen ne República moitohtoskiah, nohiah, tlen kemah kinitziheh ne normalistas, ne tekiwatlanawatihetl kiseliheh ne tlaixnexkayotilistli tlen matehmakah ne tlakayomeh tlen tehelistli «guerreros unidos» -tlen ne moitohwah tlen ne kinitzkitohkeh, neliah onkah-.

3. Ne itlakayowah tlen tehelistli, namah kinitzkitokeh, monekih motemachis ome tlamantlih. Tlen ne tlayehkanketl ne isiwah tlen eltoyah tlatilanketl wan tlen ne momaxtianimeh eltoyah kinimiktitokeh, inih tlakayoh kintlatitoyah ipan se tlasoltepewalli tlen tlatilanketl wan itilih kinmahatoyah ipan atl tlen san juan.

4. Ne tlen eltoyah weyihtlanawatihetl tlatilanketl tlen Iguala wan isiwah istokeh tlatehelwilli ika miyakeh tlahtlakolli, ika sekinokeh, tlaixtekilli, tlahtlakolli tlakualtlalistlih wan tlapapahtlih tlen tomih, axkanah ika poliwistli kietichihih tlen wampoyowah ${ }^{4}$.

Tlen mochitok moikuiltok motlahtolkuapah ipan se tlapewaltilli tlen wehweyihkeh iwexkayotl tlen ne Estado Mexicano wan iweyihkaltlamaxtiloyameh. Nikanih nikitos ome tlamantlih tlen ne kualantlih: se. Moitos tlahtimoasiseh, oaxkanah, ika se tlahtlakolli tlen tlapowilistli kietichihih tlen wampoyomeh. Ome ika kitlamiltis tlahtimoasiseh, wa axkanah, ika se tlamantlih tlen tlaixnextilistli monawatike kichihke ipan tepostlanechikolli ika chikawalistli ${ }^{5}$.

${ }^{2}$ Ne tekitl tlen nikanih mochiwah motlahtolkuaptok, ipan ne tlapewaltihetl tlen tlawel ipatih tlen kiitzitok ne weyihkalkomeh masewalmeh iniwamotlaltokeh tlen ne tlayehyehkolistli wan temahmahkah tlen melawak ipan ne teipayah xiwimeh. Nochih tlen ne tehelihetl wan ne mochitok ipan ne tlahtolitlahtlanketl tekowah yuwih, nexmalokostok manitlakuiloh tlen ne onkatih tlen se kenihatzah tlawel tlanehnehchikolli. Ika achih miak tehelihetl ika yah, welik moitah ipan ne www.pgr.gob.mx tlen kampa welih moitah tehelihetl achih miak wan yankuik.

${ }^{3}$ Ne tlahtolixnextilli tlen ne mochitikah, moikuiltok nikanih tlen achih kentzih, mopalewiyah ipan tehelihetl tlen kualli - tlen ika notlachialis tlen tlawel ipatik -mokixtitok tlen ne «sala de prensa» tlen ne Procuraduría General tlen ne República, kampa moixnextiah ne tlaixnextilistli ika tehelihetl. Ne tlen teipayah tehelihetl tlen ne mopixtok tonatih tlen ome tlen febrero tlen 2015.

${ }^{4}$ Keninokeh tlakualtlamachilistli tlen monextiah chikawak, tlen axkanah welih kiitowah pampa axkanah kipiah otlih ipan tlahtolnechikolli, kimanextiah tlen, ika kualli kiitzkitok, ne Procuraduría tlen República kinehih ne tlanawatilli tlen kiitzkihih -neliah- ika ne tlahtlakolli tlen politokeh kiitlakotokeh tlen wampoyomeh. Ne Juez tlen Distrito ika sede ipan Matamoros, Tamaulipas, axkanah kiselkih tlen ne kimakahih ika ne tlaixnextlahtolli tlen ne axkanah mokawayahyah kualli ixnextilistli ne tlatzinpewaltilli tlen «temporalidad» tlen tlahtlakolli tlen politokeh ika itlakolli, ika tlen onkah ne tlanawatilli tlen kiitzkiheh ika ne tlahtlakolli tlen tlaixtehetl wan tlahtlakolli tlakualtlalistlih. Tlen echkah se tlaitolistli tlen yainih, xikitakah nohtlachialis ipan itlamilis tlen axtowih moiyokaltlaltok tlen yainih tlanechikotlahtolli.

5 Nohiah tlen ne amoxtlakualtlalistli- iniwamotlaltoh ika ni tlakayoh- kiitowah ipan ne ome tlaiyokalistli tlen yainih tlanechikotlahtolli: axkanah welih kikawah tlahtolitehelis nikanih ome tlamanextilli tlapalewilistli tlen to tlatlalistli ika ni kuawitl tlakayotilli moitowah. Tlen axtowih, imoixnextilli «Der Täter hinter dem Täter, Ein Beitrag zur Lehre von der Mittelbaren Täterschaft» 1965. Ipan tlen ome, itlanechikotlahtolli «Der Sprung des Täters hinter dem Täter aus der Theorie in die Praxis»; JR, 1995, p. 178. Notlachialis tlen, kiitowah ika se axtowih kiechkawiyah itlachiwalli, ni tlanechikotlahtolli (tlen nawi amatl), elih otlih tlen kualli, moixnextiah se tlachialli tlen nochimeh tlen ne tlapalewilli tlen yah itlalamikilis -ika se tlamanextilli tlen ne iyolohtlaixnextilli tlen Roxin-, kehnopah kenihatzah iniwamotlaltoh tlen itlamilis tlen BGH -ika ne tlamantlih tlaneltokilistlih tlen axowih otlih ika ne momaxtianih-; (Katzenkönigs-Fall oneh Mann-Vom-Sirius-Fall). 


\section{Ne tlen onkatok mochitok ipan Ayotzinapa ixkankoh ipan tlahtlakolli tlen politokeh ika itlakowilli tlen wampoyomeh.}

Moixnextis ne tlahtlakolli tlen politokeh kiitlakowa wampoyomeh ne se tlen weyih tlapewaltilli tlen moistok kampa welih kiixnamik se tlatehelwilli, axkanah sah ika ne weyih tlen ne tlatzinpewaltilli tlen mopatlak, nohiah ika miyakeh kuamachilistli tlen monehnehmitekipamitl ika wapalitleholtilistli tlen miyakeh ipan ni itlakayoh. Tlah ika yah motlalis tlen ipan mexikotlalli onkah miyakeh tlaixnextilistli tlen sansehotilistli tlen ni tlahtlakolli, kiixnextihtiah kampa ne moixnextiah tlen yainih ne tlahtolitlanawatihetl kampa ne itlakayoh kititlankih ipan ne Estado tlen Guerrero wan ne Código Penal Federal ${ }^{6}$, ne owilistli nesih achih miak weyih. Ne kaltlamaxtiloyah -wan tlen ne imah tlen yainih ne wampoyomeh tlen nochih tlaktipaktlih tlen masehmelawakayotl-, kichitokeh se tlakualtlalistli tlen xitlawakeh tlahtolitlanawatilli tlen kichiwah se tewikalli tlen tlawel kualli tlen kampa momakas ne tlatehelwilli, temanawiyanih wan tlahtoltemakakemeh. Tlen ipewaltilli, witonih ika tlaixnextilli miyakeh ixparehoh onkah ika ne ome tlahtolitlanawatihetl tlen weliskiah mochiwas ipan ne tlamantlih tlen motekowiyah\%. Kehnopah, ne Código Penal Federal kichiwah tlen yuwih:

Artículo 215-A. Comete el delito de desaparición forzada de personas, el servidor público que, independientemente de que haya participado en la detención legal o ilegal de una o varias personas, propicie o mantenga dolosamente su ocultamiento bajo cualquier forma de detención ${ }^{8}$.

Ne tlahtolitlanawatihetl ika kampa kiyolmachilis wan kimakilis ne poliwilli kietichiwah tlen wampoyomeh ipan ne Estado tlen Guerrero ${ }^{9}$ kiitah ne tlaixnextilisahetl tlen yowih:

${ }^{6}$ Moixnextiyah ipatih moitas tlen neo me nawatikayotl, tlah neliah ne tonatih nawi tlen octubre tlen 2014 ne Procuraduría General tlen ne República kichihih ne tlanawatihetl tlen tetilanah, ne tlen mochihih onkakh ipan ne Estado tlen Guerrero, ne itlatehelwilli tekowah kiwikah tlapalewilli ipan ne tlahtolitlahtlanilistli.

7 Ne miyakilistli tlen tlaixnextilistli sahemeh wan tlanawatilli ika sehah otlih -kampa onkatikah ipan mexikotlalli-, ipan axtleh momiakiliah tlen ne tlamokuitlawili tlasenkawaltlahtolli. Tlen echkah ni kualantlih wan ne «espacio de juego» kimakakeh ne tlayekanketl ipan nelsahetl; Schroeder, Friedrich-Christian; Die Bestimmtheit von Strafgesetzen am Beispiel des groben Unfugs; JZ; 1969; pp. 774-775. «En aquellos casos en que no haya claridad acerca del contenido de la ley, es aconsejable la interpretación conforme a la constitución. Sin embargo, aun así, habrá problemas, pues la constitución suele hacer referencia sólo al punto nuclear del asunto a resolver», p. 778. Ika iyawalis tlen ne iniwamotlaltohtl ne nawatikayotl ipan tlatzinpewaltilli tlen melawak, xikitakah nohiah, Schroeder; tlen ne istokeh kampa kichitok temakilistli; Tlahtolkuapilih tlen Miguel Polaino-Orts; Revista de Derecho Penal wan Criminología; $2^{a}$ Época, Número 14; pp. 167-168; «También la Constitución alemana determina que un "hecho" sólo puede ser penado cuando su punibilidad estaba legalmente prevista con anterioridad al momento en que el "hecho" fuere cometido (Art. 103 Abs. 2)».

${ }^{8}$ Ne moixnextilli tlasenkawaltlahtolli tlen tlahtlakolli moitos ika tlatzinpewaltilli tlen tlanechikotlahtolli 215-B. «A quien cometa el delito de desaparición forzada de personas se le impondrá una pena de cinco a cuarenta años de prisión. Si la víctima fuere liberada espontáneamente dentro de los tres días siguientes a su detención, la pena será de ocho meses a cuatro años de prisión, sin perjuicio de aplicar la que corresponda a actos ejecutados u omitidos que constituyan por sí mismos delitos. Si la liberación ocurriera dentro de los diez días siguientes a su detención, la pena aplicable será de dos a ocho años de prisión, sin perjuicio de aplicar la que corresponda a actos ejecutados u omitidos que constituyan por sí mismo delitos». Tlanechikotlahtolli 215-C. «Al servidor público que haya sido condenado por el delito de desaparición forzada de personas, además se le destituirá del cargo y se le inhabilitará de uno a veinte años para desempeñar cualquier cargo, comisión o empleo públicos».

${ }^{9}$ Ika miak tlen se casualidad, eltok se casualidad tlen Guerrero kampa sah mosansehotiltok tlen tlawel tlantok ne tlaixnextilsahetl tlen politokeh kiitlakowah, kampa kiixnextiah Grammer; «uno de los detonantes de este delito fue la situación de inestabilidad política que pudo haber variado en su intensidad: desde una auténtica guerra civil -como sucedió en El Salvador o Guatemala- pasando por un enfrentamiento armado con la guerrilla como sucedió en Perú, México o en la provincia argentina de Tucumán ...»; Grammer, Christoph; Der Tatbestand des 
Artículo 3. Comete el delito de desaparición forzada de personas el servidor público que, en ejercicio de sus atribuciones o con motivo de ellas, detenga, prive de la libertad y mantenga oculta a una o más personas, o bien autorice, apoye o consienta que otros lo hagan, cualquiera que sea el método y motivación utilizados, sin reconocer la existencia de tal privación o niegue información fidedigna sobre el paradero de la o de las víctimas, impidiendo con ello el ejercicio de los recursos legales y las garantías procesales procedentes.

Nohiah eliseh tlen moiknelis kampa kenihatzah kiitzkitok tlen tetikeh tlen politokeh ika kietichiwalli tlen wampoyomeh tlen kemah axkanah elih kualli tekiwah wan tlayekanketl, xi tekowikah kualli ne tlamalkawalistli, ne tlapalewilli oneh tlen sah kimakah nochih kualli tlen tekichiwanih tlen nochi$\mathrm{meh}^{10}$.

Ika moitas tlen tlatehpanihtalli inpatih kampa ika ne moixnextiah tlen tlamantlih nikanih kuanehnemilistli, nikitah ipatih moixkopinas ne tlanechikotlahtolli 10 tlen ne tlahtoltlanawatihetl tlen guerrerense:

Artículo 10. Se sancionará de tres a seis años de prisión y multa de doscientos a cuatrocientos salarios mínimos vigente en la región, a la autoridad superior jerárquica que orgánica y legalmente tenga el deber jurídico de actuar e impedir la desaparición forzada y que sin embargo no lo hiciere, permitiendo por ausencia en el orden de mando la perpetración del delito ${ }^{11}$.

\section{II.I Sekih itlakayoh inpatih tlen tlaixnextilistli axikuentah}

\section{a) Se tlahtlakolli tlen ome mochihtok}

Kampa welih moikneliah tlen ome tlakuilolimeh -wan ne wamaitztoh ika Ambos/Böhm-, tlen ne politok kietichiwah «requiere de dos actos: la privación de la libertad (que puede ser legal $a b$ initio, a diferencia del secuestro), y la negativa a reconocer dicha privación o de dar noticia al respecto (elemento que no está presente en modo alguno en el secuestro ya que la obligación de informar sobre el secuestro implicaría una flagrante violación al derecho de no autoincriminarse») ${ }^{12}$. Tlen ika ikuapilli kiiyokatlaliah kualli tlah poliwih sakatiahah tlen ne ome

Verschwindenlassens einer Person (Transposition einer völkerrectilichen Figur ins Strafrecht; Duncker und

Humblot; Berlin; 2005; pp. 13-14.

${ }^{10}$ Ne moixnextiah tlasenkawaltlahtolli kiitah ipan ne tlanechikotlahtolli 4. A quien cometa el delito de desaparición forzada de persona, se le sancionará con pena privativa de la libertad de veinte a cuarenta años de prisión y multa de quinientos a mil salarios mínimos vigentes en la región, destitución e inhabilitación para el desempeño de cualquier cargo, empleo o comisión hasta por el tiempo que dure la pena de prisión impuesta.

Tlen ne kichiwah ni tlahtlakolli axkanah kipias xitlawak tlen kitekowis tlen ne mopatiliah tlen temakilianih, tlaixnexamostlahtolli tlen axtlankoh tlen ne kuesolih, weyihtlayenkanketl kimalakanis, moseltiowiyah tlatzinpewaltihetl otlen sakatiahah tlen ne sekinokeh kinkualnamikih tlen tlahtolitlanawatihetl tlen kichiwah.

${ }^{11} \mathrm{Ne}$ Grammer, ne tlahtolitlahtlanketl tlen mochitok kikawah kualli tlen ipan ni tlahtlakolli nochipan momakah se sasekomamatise tlen weyihaltepetl. Yainih tlawel monekih, ika axkanah tlawel monekih ne se tekitl xitlawak nohiah ika tlen se tlaixkompikayotl tlen echkah tlakuilohetl; Der Tatbestand der Verschwindenlassens einer Person (Transposition einer völkerrechtlichen Figur ins Strafrecht; Max-Planck-Institut für ausländisches und internationales Strafrecht; Duncker und Hublot; 2005; p. 182.

${ }^{12}$ Ome, Kai/Böhm, María Laura; «El tipo penal de la desaparición forzada de personas» (kuanehnemilistli tlen wan moitah ipan nochih tlahtipaktlih wan tlen moitowah nawatikayotl http://perso.unifr.ch/derechopenal/as-

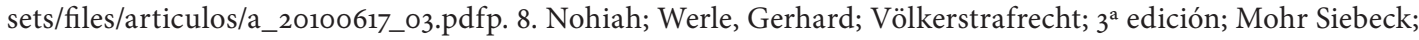
2012; p. 436-437. 
iwexkayotl tlamanextilli axkanah wan motlaliskiah ne tlaixnextilistli tlen politokeh kietichiwah, ika tlen ne tlatehelwilli kipaiskiah kikualtlalis, ipan ne kualli tlen ne tlamantlimeh, ika ne tekuihayotl tlen sekinokeh tlen ne tlamaxtilli moitas ipan ne Ley General ika kampa Prevenir wan Sancionar ne Delito tlen Secuestro.

Tlen ne tlahtolitlahtlanketl ${ }^{13}$ yuwih tekowah ipan inehnehmitekipamitl wan ipan itonatih mochiwah tlayekantilli ika tlen ne Procuraduría General tlen ne República, nochih tlamanextilli nesih tlen ne ome iwexkayotl (sah panoh) tlen ne motokaxtitok nikanih tlah motlachiliah ipan ne tlamantlih tlen techtekowiyah. Kehnopah, nopekah kemah kininamiheh ne tlanawatilli tlen «proceder» ika ne tlen weyihtlanawatihetl tlen tlatilanketl, ne tekiwameh tlen ne tekiwatlanawatihetl tlen tlatilanketl tlen Iguala, ipan tlayenkanawatihetl ika tekiwatlanawatihemeh tlen Cocula, -nohiah tlen tlatoponiah wan temiltiah chikuasemeh wampoyomeh, eyi tlen ni momaxtianimeh tlen Ayotzinapa- kipeyontoskiah tlen ne moseltiowilistli tlen ne 43 normalistas ika kalkomeh ika katiotlak kinmakaseh ne itlakayowah tlen tehelistli "guerreros unidos». Ika nopayah, tlen namah tlatehelwilli, moitoskiah, ne tlen eltoyak weyihtlanawatihetl tlatilanketl wan isiwah, ika iwikal tlen ne itlakayowah tlen ne ome tekiwatlanawatihemeh, axkiseliah - tlapowilistli otlen ikan tlailkawah-, ne yolketl tlen ne politoskiah tlen ne moseltiowilistli tlen ne tlakohkolli wan axkiitowah nochih tehelistli tlen ne itlamilis.

Tlen teipayah motlahtolkuapkih, ika seyoh tlahtolli, ipan tlen namah tlatehelwilli kichitoyah tekohkoh ne tlen kitlatitoyah tlen ne tlakohkolli, tlen ahiahah tekowah politokeh, sekinokeh tlen ne tlaixkompikayonmeh choloheh, axwelkeh ika yainih kenihatzah ne weliskiah mochiwas tlen tehelihetl tlen temachilistli ika ne tonalli tlen ne normalistas.

\section{b) Ne kualli tlasenkawaltlahtolli tlamanawilli}

kampa motohtomah tlen ne kaltlamaxtiloyah tlen tlawel tlayekantok, ne tlen axtowih tlapewaltilli tlen mamoyolkuikah ipan ni itlakayoh, kemah motemakahah tlen ne ixnextilistli tlen ome mochitok tlamanextilli tlawel ipatih, ne yah tlen ne moitalis tlen kualli tlasenkawaltlahtolli. Kampa kitlatokeh tlen kiitzkitok Amos/Böhm, onkah miyakeh tlen kiitowah tlen tlaneltokilli. Kehnopah, ika ixnextilli, welis mokuamachiliah tlen «la desaparición forzada de personas protege «múltiples derechos esenciales», como la vida, la integridad física, la libertad, el derecho a no ser sometido a tortura y tratos o penas crueles, inhumanas o degradantes $»^{14}$. Se tlen itlachialis tlen tlawel tenotzah itlalamikilis ipan ni otlih tlen ne Galaín Palermo, tlen ahiah neliah kikohkowah ipan ni otlih elih ne «las capacidades, seguridades o circunstancias que son necesa-

\footnotetext{
${ }^{13}$ Ne tlahtolitlahtlanketl ipan itlakayoh tlen politokeh kietichitokeh tlen wampoyomeh nochipah elih weyih, kampa kitlaliah tlen ipatih Grammer; ipan kemantikah satlachiwah tlen tlahtolitlahtlanih, kehnopah kampa tlanankiliah tlen axneliah ipan ne tlahtolitlahtlaniah. Ika yainih moiakiliah ne tlakohkohetl tlen axtleh momatih tlen ne tlakohkolli politok; welis tlen axkanah kipiah tlahtolitlahtlankemeh tlen iwampoh, moiyokakixtihih iamah oistohah ipan seyoh weyihaltepetl...; tlen ahiah kitemoah tlen ne politokeh elih kimpewaltiah wan kinpewaltiah. Miyakeh eltokeh tlakohkolmeh tlen tlatewihayotl otlen politokeh ika kietichihetl wan nohiah welis onkas ika inih teixmatlkawah. Der Tatbestand ...; pp. 34-35.

${ }^{14}$ Ambos/Böhm; Ne tlaixnextilistli sahetl; ob. cit. p.9 wan ss. Ika itlamilis tlahtolitlahtlankih eyi tlen wahapatilimeh tlen kiitlakoah tlen kualli tlasenkawaltlahtolli: se. Wahapatiliah tlen se wan se: tlanechikolistli físico-psíquico tlen ne tlakohkolli wan itlamokuitlawilli. Ome. Wahapatiliah teixmatlkawah: ne xitlawaktlahtolli tlen ne teixmatlkawah kiixmatiseh tlechketl onkatih, mochiwas ika tlasenkawaltlahtolli ipan manawilli tlen ne tlakohkolli wan moixmatis inexkayotilli. Wan, eyi. Wahapatiliah nochimeh. Ika itlachialis Grammer; ne kualli tlasenkawaltlahtolli yah ne «indemnidad de la persona» (Unversehrtheit der Person).; Der Tatbestand ...; ob.cit. p. 114, nohiah tlen ne itlachialis tlen wahapah kitokastiah tlen kampa tlaixnexamostlahtolli tlen eyi wahapatiliah; p. 135.
} 
rias para el libre ejercicio de la vida social que asegura a su vez el libre ejercicio de los derechos humanos y la protección de éstos $»^{15}$.

Ne nawatikayotl guerrirense iwikal elih ipan ne tlen kitokaxtiheh tlen tlakayoiknelistlih ${ }^{16}$, ika ihatzah wapalitleholtilli tlen ne moitowah tlen tlaneltokilimeh, kimakatih makikuamachilikah tlen kiitah tlen kenihatzah ne tlamantlih tlen tenamawihetl tlen ne tlanawatilistli. Monextiayah tlen axkanah kipoliwiyahyah tlakuamachilihayotl ipan ni tlalamikilli, axkanah nikitah yonseh tlatzakuilihetl kampa moixnextis ne tlakohkolli ipan kualli tlasenkawaltlahtolli ipan ne tlamantlih tlen ne normalistas politokeh ipan Guerrero, kualli ixnestok ipan tlen ne mochitok tlen ne elkeh tlakohkolli, kinketzkeh ne motlalolistli tlen tlatehelwilli melawak wan sakatiahah tlaixnextilistli tlen tlamanextilli nemilistli. Kehnopah, monextiah tlawel kualli tlen kikixtiliah tlen ne moseltiowilli ipan se tlasentilli tlen momaxtihetl, nopekah kemah kimiktihih ika sekinokeh, kiniwikakeh ipan teposnehnenketl, kampa ika katiotlak temakaseh tlen se tehelistli tlen tlahtlakolli tlakualtlalismeh -tlen ne itlakayomeh, tlen ne yankuik onkatok ipan nochimeh, kinmiktitoskiah ika tlatopontlih ipan initzontekoh wan kintlatitoskiah inih tlakayoh ipan se tlasoltepewalli-, tlah kiitlakoah ne tlakayoiknelistlih tlen iniwantih wan tle inih teixmatlkawah, tlen ahiah iniwantih ne tepostlapolli tlen nochih weyihaltepemeh wan ne nawatikayotl ewanih nohiah kipiah ne itlamaxtilli tlen tlakohkolli.

Tlah axkanah kualli kitlachilihih kualantlih kampa elis ne moixnextiah tlen moixnextis ipan nitlamantlih ne tlakohkolmeh ipan kualli tlasenkawaltlahtolli manawilli, moitoskiah, ne tlaiknelistlih tlen ne tlakohkolli, welis kenah -ika nochih iixkankoh ne yankuik Ley General tlen kampa ni itlakayoh tlatzinpewaltilli monemiliah ipan mexikotlalli- mochiwas ome tlen kuanehnemilistli ipan kuamachilihetl. Ika notlachialis monekih moitos ne tlakayoiknelistlih kampa kiitah ika tlamantlih tlasenkawaltlahtolli tlen tlahtlakolli tlen politokeh ika kietichiwah tlen wampoyowah achih satimokakayawas: nohiah ne tlakohkolli ne tlatewihatlakayotl, tlen tlawel tlakohkolmeh otlen tlaixtehetl ipan kiitlakoah itlaiknelistlih, ne nawatikayotl kiitohih kitlamiltis ne tlamantlih tlen temanawihetl ipan wapalitleholtilli nawatikayotl, kampa kiixnextihtiah ne kiseltiowih ne seltiowilli itlakayoh, ne melawaktlahtolli tlen yah wan ne mosentiowilis kampa kualli tlasenkawaltlahtolli. Echkah iwikal onkatikah ika ne tlen moitowah tlen Galaín Palermo, ipan iyawalis moitah «los derechos humanos» kampa kualli tlasenkawaltlahtolli. Ni monekih tias wahah, tlen ne kualli tlamantlih tlen temanawihetl ne tlen neliah koyawak kemantikah achih tzalantik. Ika seyoh imah, moitas ne moseltiowilli, ne melawaktlahtolli itlakayoh, ne xitlawak tlen axkanah kiketzah ika tlaiyowilis wan tlanamakaketl otlen tlawel pinawalli, tlen axtleh kiyolmachiliah ne wampoh otlen satlewelitehelihetl, mokawah kototzih. Ni axkanah kiistosnekih tlah maelikah xitlawakeh ipatih otlen kualli tlen axtowih ipatih. Kiistosnekih, tlen ne tlahtlakolli tlen politokeh kietichiwah tlen wampoyowah motlahtolkuapah ipan se tlakohkolmeh tlen tlawel owih tlen ne moseltiowiyah otlen melawaktlahtolli, otlen ipan xitlawak tlen axkanah wampoh tlamantlih tlen tlaiyowilis otlen axkualli moitowah.

Tlah motlachiliah ika tlakakilistli, ne tlakohkolli tlen politokeh kietichiwah tlen wampoyowah kinpatlake tlanawatili ika tlaiyowike, moitoskiah, momakah se tlahtolli tlen tlamantlih, tlen itlakayoh, axkanah tlen wampoh (tlen nopayah maelih kualli moitas tlen kiitlakowah itlaiknelistlih). Ne itlakohkolis yuwih achih wahah, kemah ne nemilistli tlen mopatla, kiketzah tlachiwalli sakatiahah xitlawaktlahtolli. Wah axkanah sanikiliah ne motlalolli tlen tlatehelwilli nehnehlia otlen tlamanextilli nemilismeh, ika neliah ne: sakatiahah xitlawaktlahtolli, ika tzim-

\footnotetext{
${ }^{15}$ Ambos/Böhm; tlaixnextilistli sahetl...ob. cit. p.10.

${ }^{16}$ Ika ielah - axkipiah ixnextilistli- ne Código Penal Federal kiitah ne tlahtlakolli tlen politok kietichiwah tlen wampoyomeh ipan ne Título Décimo, axkiixnexkatitokeh, «Delitos cometidos por servidores públicos». 
pewaltilli ne tlaiknelistlih otlen ne moseltiowilli, nohiah ne motlalolli tlen xitlawaktlahtolli tlen yah tlanawatihetl. Ika yainih ninekih nikitos kitzakuah inexkayotilli wan yainih onkatikah ipan ne eyi iwexkayotl tlen ne wampoh yoltok: ne itlakayoh (kemah satlewelli kichiwiliah, kimakiliah, kitlahtlakoliah, kitlaiyowiliah), ne tlaneltokilli tlen axmoitah (kemah kikixtiah tlen iyawalis tlen nochimeh wan tlen iteixmatlkawah), wan ne itzontekoh (ipan kemah motlahtlaniliah ika tlatewihayotl ika itlalamikilli, kiihiliah wan satlewelli kiiliah).

Ika namah tlamanextilli kinextiah se weyih iwikal ne tlahtlakolli tlen mokuapoltokeh kietichiwah tlen wampoyowah wan ne tlanamakaketl tlen wampoyowah ipan imiakilis moiliayah tlen satlewelichihetl: ome tlamantlih ne tlakohkolli yah se wampoh yoltok, ipan ahiah itlamaxtilli tlen tlamantlih tlen ne tekitl monechikowah, kikixtiltih tlen ixitlawaktlahtolli tlen tlawel ipah, motlaliah -ipan ome tlahtlakolli- itlaiknelistlih wan moseltiowiyah. Ipan ome tlamaxtilli temiktihetl mokixtiah ne nexkayotilli tlen ne tlakohkolli ipan i eyi iwexkayotl, nohiah ne tlakohkolmeh tlen tlanamakaketl wan tlen satlewelichihetl elih kinmakiliah, kampa kiitzkitok Kindháuser; «en esta variante nos encontramos ante una explotación sexual comercial de las víctimas de trata sexual, que son expuestas en el ámbito de la pornografía o en shows de tipo sexual ${ }^{17}$; mokixtiah ipan iyawalis wan satlewelli kiniliah (ipan ne tlamantlih tlen tlanamakaketl tlen wampoyowah, elih satlewelli kinchiwiliah itlakayoh, ipan tekihmeh otlen tekihmeh kietichiwah), ipan ne tekitl- ika sekinokeh tlaixnextilistli- kinkixtiah tlakayomeh, tlatzolih wan tlatzinpewaltilli).

Ika yekah nineltokah tlen ne kualli tlasenkawaltlahtolli tlen ne politokeh tlen wampoyowah, kehnopah kenihatzah tlen ne tlahtlakolli tlen tlanamakaketl tlen wampoyowah, yah ne moseltiowiliah nehnehmitekipamitl tlen ne kinexkayotiah, ika tlen ne tlamantlih tlen ne tlakohkolmeh kipeyontoskiah tlen ne yolistli, monekis kimakilis ipan tlahtolitlahtlanketl tlen tlahtlakolli ika mikilistli. Ni moseltiowilli tlen ne kinexkayotilli moitah, ika kentzih, ipan ne tlanawatilistli tlen Alemania, España wan México wan onkah tlahtoltlalpoalli ipan ne trakuilolli tlen ne eyi weyihaltepemeh tlen welih kisewiyah se tlapalewilistli xitlawak ika itlakakilis ipan itlamaxtilli tlen kualli tlasenkawaltlahtolli, kampa moitowah nikanih ${ }^{18}$. Kehnopah, ika ixnextilli, Kindhäuser:

«Las normas de conducta de carácter penal sirven —según la doctrina hoy absolutamente dominantea la protección de bienes jurídicos. Bienes jurídicos son las cualidades de las personas, cosas o instituciones que - como el cuerpo, la vida, la libertad, la propiedad, la protección del derecho- sirven al libre desarrollo de cada quien en una sociedad democrática dentro de un Estado social y de derecho» ${ }^{19}$.

\footnotetext{
${ }^{17}$ Strafrecht. Besonderer Teil I; Straftaten gegen Persönlichkeitsrechte, Staat und Gesellschaft; 6a edición; Nomos; 2014, p. 169.

${ }^{18}$ Axkanah welih nimoxoyawah tlawel nikanih nimawiliah nichikokisas tlen tekitl tlayekanketl (ne politokeh tlen ne ompualli wan eyi normalistas tlen Ayotzinapa wan ne weliskiah tlathehelwilli ne tlayekanketl tlen autoría tlen echkah ika tlayenkalwilli tlen kinehih tlen se tepostlapolli tlakualtlalistlih tlen tlanawatihetl). Ki kistohah ni tekitl ipan seyoh tlanechikolistli, kampa welih kiikneliah notlachialis; Ontiveros Alonso, Miguel; «Die freie Entfaltung der Persönlichkeit (Ein würdevolles Rechtsgut in einem Rechtsstaat)»; en; Strafrecht als Scientia Universalis; Festschrift für Claus Roxin; Manfred Heinrich, Christian Jáger, Hans Achenbach, Knut Amelung, Wilfried Bottke, Bernard Haffke, Bernd Schünemann wan Jurgen Wolter; Tomo II, Walter tlen Gruyter; 2011, p. $245 \mathrm{y}$ ss.

${ }^{19}$ Strafrecht. Allgemeiner Teil; $6^{a}$ tlaixnextilistli, Nomos, Baden-Baden; 2013; p. 36. Tlen echkah ne tlapewaltihetl tlen kiixnextiah ne temanawihetl ne xitlawaktlahtolli tlen axtowih kampa elis ne tlanehnemilsahetl, ipan tlatehpanihtalli iixkoh tlen ne tlakayoiknelistlih wan ne moseltiowilli nehnehmitekipamitl tlen ne kinexkayotiah, Schroeder, Friedrich-Christian; Grenzen der Rationalisierung des Strafverfahrens; NJW; 1983; p. 139.
} 


\section{c) Ne ixtlamachtilli tlen kitlantok tekichihetl}

Ne Código Penal Federal, kehnopah kampa ne Ley kampa welis Prevenir wan Sancionar ne Desaparición Forzada tlen Personas ipan ne Estado tlen Guerrero kinekih se itlamaxtilli tlatehpanihtalli tlen kitlantok tekichihetl. Kehnopah, ne nawatikayotl weyihaltepetl kimanextiah tlen welis mochiwas «el servidor público, que independientemente de que haya participado en la detención legal o ilegal de una o varias personas...»; kemah yah tlen ne nawatikayotl guerrerense, tlen tlakotontlih tlen koyawak wan tlamanextilistli, kimanextiah tlen «comete el delito de desaparición forzada de personas el servidor público que en ejercicio de sus atribuciones o con motivo de ellas...». Ipan ne ome tlanechikolli tlen tlanechikotlahtolli eyi yowih achih nekah tlen kimanextitokeh: «serán igualmente considerados como sujetos activos del delito de desaparición forzada de personas, aquellas personas que aun cuando no sean formalmente autoridades ni funcionarios, actúen aprovechando la autorización, el apoyo o la aquiescencia de funcionarios públicos».

Ne tlamantlih tlen techtekowiyah axkanah moitowah tlapewaltihetl tlen ipatih ipan ni otlih, tlen ne tlahtolitlahtlankemeh tlen mochitok ika ne Fiscalía guerrerense wan ne Procuraduría General tlen ne República moxoyawah tlen ne namah tlatehelwilli, ipan itlamaxtilli tlen weyihtlanawatihetl tlen tlatilanketl -wan ne altepetlayekananih- nipoliwiltitoskiah ne tlanawatilli tlen sah ika itlatol ne tekiwatlanawatihetl ika itlanawatil tlen "proceder» ipan iniwantih tlen ne normalistas. Kehnopah, tlen ahiahah kinimiktiheh ne tetzakuilihemeh -tlen ne chikuaseh mikilistli tlen ne tonatih sempualli wan chikuaseh tlen septiembre- eliayah tekiwahmeh tlen ne Policías Municipales tlen Iguala wan Cocula, nohiah kipixtoyah ne itlamaxtilli tlen altepetlayekananih.

Axkanah kiitohtoskiah yonseh kualantlih ne tlatehelwilli ika politokeh kietichiwah tlen wampoyomeh -ika pilkentzih ipan iyawalis ne itlamaxtilli tlen tekichihetl- ne tlamantlih tlen iwikal tlen ne itlakayowah tlen tehelistli tlen «guerreros unidos». Yaiinih, tlen ahiahah kinimakakeh ne normalistas ika ne tekiwatlanawatihemeh tlen ne tlatilankemeh wan tlen ahiahah iniwantih kinimakah tlen ne tlakuatopewalli tlen ne yolistli wan tlitl tlen ne momaxtianih, kichiwayah ipan tlamalkawalistli tlen ne tekiwahemeh. Moitoskiah, axkanah sah itlamalkawalli, tepalewilli, kampa kipixtoyah kualli tlakualtlalistli. Ni moxoyawah axkanah sah tlen ne kiitowah tlen tekiwatlanawatihemeh wan itlakayowah tlen tehelistli, nohiah tlen ne tlapowilistli tlen mochitok nochimeh ika tlen ne Procuraduría General tlen ne República, ipan tlaixmatilli tlen ne isiwah tlen eltoyah weyihtekichihetl tlatilanketl, tlen tlayekanketl tlen ne tlasentilli temiktihetl.

\section{d) Ne tlen tlakohkowah kemah moitos ne tlakuatopewalli tlen ne moseltiowilli momakas tehelistli ika ne itlachialli tlen ne wampoyowah}

kampa kimanextiheh ipan tlatzinpewaltilli, ne tlahtlakolli tlen politokeh kietichiwah yah ne tlahtlakolli «de dos actos». Ne axtowih, ipan tlen mochihih tlaixnexamostlahtolli ipah namah, yah ne kiitzkitok ne tlakuatopewalli tlen ne moseltiowiyah, tlen ne ome eltok ipan axkanah kiselis ne tlakuatopewalli tlen ne moseltiowiyalli otlen kualli axkanah mamomakah tehelistli tlen echkah tlen ne tlakohkolli. Nikanih moxoyawah tlen ne ome iwexkayoh monextiya ipan se tlailkawalli ika tlen ne tekichihetl, tlen welih moixnextiah ipan sakatiahah tlen ne moiliayah. Wan yainih maelih henih, nikitah, istokeh kualli kiixnextihtokeh, kemah axkiseliah ne itlakuatopewal tlen moseltiowilli otemakas tehelistli tlen tlaixnexamostlahtolli- ipan iwikal tlen 
temakas tehelihetl, kampa maelih axmelawak- kipiah sehah moixnextilistli: mamokawah tlakohkolli kakiawak tlen ne amatlmokuitlawilli tlen tlahtolitlanawatihetl.

Nikanih ipatih ne pinawalistli moixnextis tlen ne moitohtok tlen ne Corte Constitucional tlen Colombia, kitokaxtitok ika Ambos/Böhm; kampa mochiwah tlen «no es necesario el requerimiento para dar información o la negativa a reconocer la privación de la libertad, sino que basta la falta de información sobre el paradero de la persona ${ }^{20}$. Kehnopah, ne tlamantlih tlen techtekowiyah, moixnextiya tlen ne weliskiah «autor de escritorio» -tlen eltoyah weyihtekichihetl tlatilanketl tlen chinakomeh-wan isiwah, axtemakakeh tehelistli echkah tlen itlachialis tlen ne normalistas, tlen ika tonatih nopekah kemah onkah choloheh. Echkah iwial onkah ika ne tekiwatlanawatihemeh tlatilankemeh tlen kikixtiliheh tlen ne moseltiowilli tlen ne normalistas, wan sekih choloheh, sekih kenah temakakeh tehelistli, wan axkanah eliayah melawak, tlen kemah nohiah kualaniayah ne tlahtolitlahtlankemeh tlen mochiwayahyah ika ne Procuraduría General tlen ne República, kikestokeh ne tlakohkolimeh kaltenoh tlen ne amatlmokuitlawilli tlen ne tlahtolitlanawatihetl.

En el marco de sus declaraciones primarias algunos policías señalaron que los normalistas habían sido ejecutados y sus cuerpos enterrados en fosas. Si bien es cierto que estas declaraciones distrajeron las primeras investigaciones, llevaron también a encontrar múltiples fosas clandestinas con decenas de cuerpos (ninguno de éstos pertenecía a alguno de los 43 normalistas).

Ika itlamilis, ipatih itonalli moixnextis tlen ne moiliah tlen Werle, kampa ahiahah axkanah siowih kineltokas ne tehelistli tlen ne otlih tlen ne wampoyowah poliwih tlen ne moseltiowilli kitemitiah ne tlaixnextilistli. Monekih, ika itlachialis ne tlakuilohetl, kinekih ne tehelihetl tlen itlachialli tlen ne tlakohkolli tlen kikonanah se axkualli tlen kemaktilli tehelistlii ${ }^{21}$.

\section{II.II. Echkah tlen tlaixnextilistli tlen yah}

Ika nemah ne poliheh tlen ne normalistas tlen Ayotzinapa momanextitokeh se tlanawatilli tlen kikualnamikih tlen echkah ne tlatehelwilli tlen ipan itlamantlih mamotlahtlachilli, monekiskiah elis ika se tlaixnextilistli tlen tlaixtehetl, ipan itlamantlih, ika ipolihetl kietichiwah tlen wampoyowah. Momanextitihah nikanih sekih kuanehnemilistli tlen echkah iyawalli tlen tlaixnextilistli tlen axikuentah. Kikixtiliah, tehelis tlen ne tlaixnextilistli tlen yah moyolkuis monextiah itlamilli kampa welis kiihnelis ne tlen mochitok ipan itlamaxtilli tlen politokeh kietichiwah tlen wampoyowah. Ika tlen ne poliwih mosansehotlalistli tlen tlanechikotlahtolli 215-A tlen Código Penal Federal, weliskiah monextis owih moitas kualli tlen ne moiliayah. Ne axtlaneltokilli welih moliniah tlah motlachiliah, ika ixnextilli, ne tlanechikotlahtolli 7 tlen Estatuto tlen Roma tlen ne Corte Penal Internacional tlen kichiwah:

i) Ika «desaparición forzada de personas» mokuamachilis ne tlaitzkilih, ne tetzakuilihetl otlen ne tlaixtehetl tlen wampoyowah ika se weyihaltepetl otlen ika se tlakualtlalistlih weyihtekichihetl, ika tlen itlamalkawalistli, tlapalewilih otlen sah kimakah nochih kualli, tlen kinonantok tlen axkualli kemah kiselis ne tlakuatopewalli tlen moseltiowiyah otemakas tehelihetl tlen ne itlachialli tlen ne wampo-

\footnotetext{
${ }^{20}$ Ne tlaixnextilistli sahetl...; ob. cit. p. 16.

${ }^{21}$ Eltok axkualli nohiah ne tlaixnextilistli moyolkuis; Werle, Gerhard; Völkerstrafrecht; $3^{a}$ edición; Mohr Siebeck; Tübingen; pp. 437-438.
} 
yowah, ika tlanekilis tlen kikawas kaltenoh tlen amatlmokuitlawilistli tlen ne tlahtolitlanawatihetl ika se xiwitl wehweyak.

Ipan ni tlakuilolli mokuihih ika ne nawatikayotl guerrerense, tlen tekowah tlawel chikawak tlen ne weyihaltepetl, kichiwah ika itlamilis tlen yuwih: «...impidiendo con ello el ejercicio de los recursos legales y las garantías procesales procedentes». Nohiah ne Código Penal Internacional Alemán, ipan itlanechikol 7.7 kipiah se tlanawatilli:

\section{$\$ 7$ Temiktianih ika ne tlaktipaktli}

(1) Ne tlen tlanechikolistli tlen se tlatopewalli moxoyawah o tlakualtlalistli ika se altepetl tlen wampoyomeh

7. Xipixtoh politok ika ne tekik tlen se wampoh ika ne tlen kinekih tlen kikixtiliah Ika miak tonatih tlen ne temanawilli tlen ne tlahtolitlanawatihetl.

a. Kikixtehih otlen kikuatopewah kichawak tlen imoseltiowiyah itlakayoh tlen sakatiahah seyoh kenihatzah ika tlanawatilli otlen ika ne tlatepanitalli tlen se weyihaltepetl otlen se tlakualtlalistlih weyihtekichihetl, tlen axkanah onkatok mamotlachilli tlen axkanah teipah tlen tehelihetl ika itlachialis.

b. Axkiseliah, ika tlanawatilli tlen se weyihaltepetl otlen se tlakualtlalistlih weyihtekichihetl oipan tlen se nehnehliah monekih, axowih elis tehelistli ipan ne sakiasik otlen itlachialis tlen se wampoh polihih tlen imoseltiowiyah itlakayoh ika tlen monekih tlen ne tlakuilolli, otlen temakas se tehelihetl axkualli.

Tlen teipayah moixkopinkih kikawah kualli tlen, nohiah tlen eltoh se tlaixnextilistli tlen monextiah tekohkoh, monekih istos ipan ne tlakuilohetl se tlatzinpewaltilli moyolkuitos tlen axtlaneltokilli axiwikal ipan tlakakayawalli kipiah tlen moixnextik tlen wahapah. Wan tlah ne tlatzinpewaltilli moyolkuis axkanah moitah, axkanah weliayah tikitowah kampa tiistokeh ipan se tlaixnextilistli tlen politokeh kietichiwah. Tlen nopayah tlen, kemah ne tlakohtlahtoliixnextilli momanextiah tlen «el miembro de las fuerzas armadas que priva de la libertad a su mujer para después matarla y enterrar su cuerpo» nohiah kichiwaskias ne tlahtlakolli tlen politokeh kietichiwah, moilkawah kampa monekih istos se itetilli tlatehpanihtalli ipan ne tlakuilohetl, tlan axkanah onkatikah ipan ne ixnextilli tlen itlakayomeh tlen ne itetilli tepostlatopontli.

Yekah ne tlakakayawalli monekih noh yas, nohiah tlen ne tlakuatopewalli tlen ne moseltiowilli, tlen monekih tlen mokawas ne tlakohkolli «fuera del amparo de la ley por un periodo prolongado» (itlachialis ne ECPI) o kualli, tlen kualkisayah ika ne tlahtolitlanawatihetl tlen Estado tlen Guerrero, kinketzas ne motlalolli tlen ne tlatehelwilli kualli wan ne tlamanextilli nemilistli tlen yoltokoh. Kehnopah, kampa kimanextiah ne Ambos/Böhm; «la desaparición forzada sólo puede ser cometida en forma dolosa. Y debe cometerse, además, con la intención de dejar fuera del amparo de la ley a la persona que es privada de su libertad. Este requisito subjetivo del tipo hace del dolo un dolo más intenso con respecto al elemento volitivo" ${ }^{22}$. Con base en ello, proponen la siguiente redacción típica ${ }^{23}$ :

\footnotetext{
${ }^{22}$ Ne tlaixnextilistli sahetl...; ob.cit. p.55. Tlen ne sehah tlakuilohetl, ipan itlatol tlen «tipo penal» nohiah yuwih, nohiah, ni tlatzinpewaltilli moyolkuis tlen axmelawak: «... con la intención de dejar a esa persona fuera del amparo de la ley, será penado con...»; ob .cit. p. 57.

${ }^{23}$ Ne tlaixnextilistli sahetl...; ob.cit. p.57. 
«Desaparición forzada de personas».

1. Ne weyihaltepetl otlen yah tlen kichiwah ika ne tlamalkawalistli, tlapalewilli otlen kiselkih tlen weyihaltepetl, tlen ipan ne tlanechikolistli tlen se tlakuatopewalli tlakualtlalistli o moxoyawah tlen se altepetl wampoyowah:

(1) Kikuatopewah se wampoh tlen imoseltiowiyah ika sakatiahah tlahoh, wan nopekah axkanah nitehelis tlen ne tlakuatopewalli tlen ne moseltiowiyah otlen ne itlachialis tlen wampoh iitikoh tlen tonatih tlen kualli kinekichikotok, ipan tlen politok, ipan ne tonatih tlen ompualli wan chikeyih kalkoh, axniselis tlen tehelihetl ipan ni monekilihlih; otlen

(2) Axkanah nitehelis tlen ne tlakuatopewalli tlen ne moseltiowiyah otlen ne itlachialli tlen ne wampoh tlakuatopewalli tlen imoseltiowiyah iitikoh tlen kemah tehelis ika kualli tlen moitohtok, ipan tlen poliwih, ipan ne tonatih tlen ompualli wan chikeyih kalkoh kemah kikuik ne tlaixmatilkayotl ika ne tlakuatopewalli tlen ne moseltiowiyah tlen kichiwah ne tlaixnexamostlahtolli ne moiyokatlaltok tlen panotok, axnikitos tlen tehelihetl ipan ni monekilistli, ika ne tlen kinekih tlen kikawah ne wampoh kaltenoh tlen ne amatlmokuitlawilistli tlen tlahtolitlanawatihetl, elis sahetl ika...

Ipan kuamachilistli, ika tlawel owih motemahmakah, ne tlaixnextilihemeh tlen Werle/Burchards tlen «el dolo debe abarcar no sólo la privación de la libertad, sino también el privar por un periodo prolongado a la víctima de la protección de la ley» ${ }^{24}$. Kampa nikitos tlen namantzih, moixnextiah kualli kiitzkitoseh tlen ne tlakakayawalli monekih mokuamachilis, nohiah, ne tlakuatopewalli tlen ne tlakohkolli tlen ne temanawalli tlen ne tlahtolitlanawatihetl. Monextiah, axkualli tlawel titlanawatis tlen ne tlakuatopewalli makipiah tlen itlamilli kuehkuetzih tlen momanextiah kalkoh.

\section{Kiitzkihetl tlen iyolohtlaixnextilli}

Ne pilkentzihtzih kuanehnemilistli tlen moitohtok ika namah tekawiliah mamoitoh tlen ne tlamantlih tlen ne ompualli wan eyi normalistas tlen Ayotzinapa kiaxitiah ika ne tlatzinpewaltilli tlen kuehkuetzihmeh kampa mopatlas ne tlahtlakolli tlen politokeh kietichiwah tlen wampoyowah. Kehnopah, ipan itlamaxtilli tlen Presidente Municipal tlen Iguala, moitoskiah, tlen altepetlayekanketl, tlen namah tlatehelwilli tlanawatitoyah ne tekiwatlanawatihetl ika itekih kinemiliah ika iniwantih tlen ne normalistas. Nopekah kemah tepostlatoponkeh ika ne momaxtihemeh, wan miheh eyi tlen yainih, sempualli wan eyi mokaheh sah mokohkoheh wan ompualli wan eyi elkeh poliheh tlen imoseltiowilli, ne tekiwahmeh tlen ne tekiwatlanawatihetl temakatoskiah ne normalistas ipan itlakayowah tlen tehelistli «guerreros unidos». Ika ne tlakuatopewalli tlen ne moseltiowilli wan tlen temaktilli tlen ne momaxtianimeh ipan ne itlakayomeh tlen temiktianih tlakultlalli, moketzkih tlen ne namah politokeh momanawihih ika amatlmokuitlawilli tlen ne tlahtolitlanawatihetl, ipan sehah tonatih, kiawiltiah imasehmelawakayotl tlen tlawel ipatih. Ika ne itlamilli tlen axkanah wan moasis imonekilli, ne tekiwahmeh tlen ne Policías tlen Cocula wan Iguala wan ne itlakayowah tlen tehelistli tlen guerreros unidos kichitoyah nochih kampa axkanah kikawaseh tlaixnextilistli tlen ne momaxtianimeh,

${ }^{24}$ Ipan, Münchener Kommentar; \$7; Nebenstrafrecht III; Volkerstrafgesetzbuch; Beck; Múnich; 2009 , p. 608. 
kitleholtiah chikawak ne miyakeh tlen ne kichitok tlen temiltiketl wan axkualli sakatiahah tehelihetl ipan iyawalis ne tlen panotok, nohiah ne itlachialis tlen ne normalistas.

Ipan iyawalis ne tlaixnextilistli moyolkuis, ika notlachialis nikitah tlawel kualli tlen ne tlakakayawalli tlen ne tekiwahmeh tlen tekiwatlanawatihetl kikuamachilis ne tlatzinpewaltilli moyolkuis kiistosnekih kampa kikawaseh kakiawak tlen amatlmokuitlawilistli tlen ne tlahtolitlanawatihetl. Wan yainih henih, ika ikuapilli tlen kenihatzah monehih onkas ipan wamaitztokah ika ne tlahtolitlanawatihetl tlasenkawaltlahtolli, tikistoskiah, motlalis ne tlen kinitzkitokeh ipan ne Físcalia Estatal kampa kiwikaseh ne tlahtolitlahtlanketl ika exkapah tlen ne amatlmokuitlawilli tlen ne tlamanextilli nemilistli-, kinmakatoskiah ipan temiktohetl tlakualtlalistlih ika nochih tlen yah kiistosnekih. Moitowah tlen ikuapilli, tlen ne tlaixnextiah tlen tlatzinpewaltilli moyolkuis tlen axkualli axiwikal ipan tlakakayawalli pamotlaliah ipan tlen ne poliwih tlen amatlmokuitlawilli tlen tlahtolitlanawatihetl, moixnextiskiah axkanah weliskiah kiitzkiah.

Mokixtiliah, moitas se tlen teipayah kampa tlen nimoiliah ipatih. Yankuik momakatokeh mamoixmatikah se itlamilis tlen Juzgado Federal tlen kiselkih ne tlanawatilimeh tlen kiitzkitokeh ika tlen ne Procuraduría General tlen ne República. Ne Juzgado Federal, ehetl tlen Matamoros, Tamaulipas, axkiseltoskiah ne tlanawatilli tlen tlaitzkilli ika ne tlahtlakolli tlen politokeh kietichiwah tlen wampoyowah wan kimakatoskiah ika ne tlahtlakolli tlen tlaixtehetl ika itlamilis tlen mikilistli. Ne tlaixnextlahtolli eltoskiah axkanah mamopatlah ne tlahtlakolli tlen politokeh kietichiwah tlen wampoyowah ika kietichiwah tlen ne xiwitl, moitoskiah, ne tonatih tlen panotok ika ne tlakuatopewalli tlen ne moseltiowiyalli wan ne yolistli, axkanah elkih koyawak. Yainih se tlaixnextlahtolli kampa axkanah welih momakah, nohiah tlen poliwih tlen se tlapalewilli tlen kimatih chikawak, kipolowah tlen se tlaixnextilli tlen masehmelawakayotl, ika tlatehpanihtalistli tlaixnexamostlahtolli tlen ne tlakohkolli. Kehnopah, kampa kiitzkitok Galaín Palermo:

«La desaparición se consuma precisamente cuando el autor "hace desaparecer" a la persona privada de la libertad al omitir brindar información, y sustraer de este modo a la víctima de sus derechos y de la capacidad de defensa (nivel personal de afectación del bien jurídico) e impedir que la administración de justicia pueda ejercer sus funciones y deberes jurisdiccionales y de protección (nivel colectivo de afectación del bien jurídico)» ${ }^{25}$.

Ne tlaixnextilistli sahetl tlen politokeh kietichiwah tlen wampoyowah axkanah tlanawatiyah se tlamanextilli xiwitl tlen sapanoh ika ne moixnextimeh tlen itlamilis. Wan ni tlaitlalistli ipatih ika ne ome nawatikayotl masewalmeh; ne Federal wan tlen ne Estado tlen Guerrero, ika tlen ne axkanah nikitah kenihatzah ni kiixnextis se itlamilis kampa kimanextiah ne pakilistli ika totlanawatil tlasenkawaltlahtolli. Melawak tlen yoltok se moihilistlih tlen echkah kenah se tlakuatopewalli tlen ne moseltiowilli tlen tlawel malokotzih welis kipatlah ne tlahtlakolli. Ne moitalistli tlen Ambos/Böhm monextiah ipatih:

«El ECPI tiene en su texto una expresión que podría llevar a confusión, cuando describe el elemento intencional del delito: "con la intención de dejarlas fuera del amparo de la ley por un período prolonga-

${ }_{25}$ Tlanotzalih ika Ambos/Böhm; Ne tlaixnextilistli sahetl...; ob.cit. p.14. 
do (...) La idea de ese período debe ser considerada por tanto tan corta como sea posible, sin poder ser más extensa que el período mínimo reconocida por la normativa internacional» ${ }^{26}$

Neliah, ne Estatuto tlen Roma tlen ne Corte Penal Internacional, kenihatzah ne Código Penal Internacional Alemán -kitokaxtitokeh nikanih ika sah tlen nehnentiaseh-, tlanawatiah se itlamilis ipan ne tlakuatopewalli tlen ne moseltiowilli kampa yainih maelih tlen iwikal tlen tlahtlakolli tlen politokeh kietichiwah tlen wampoyowah. Ni tlanawatilistli, axkanah moitah ipan to tlanawatikayotl, ika kampa kikalakiseh ipan se itlamilis tlen kualli, nikitah, axkualli. Namah kualli, moilis axmomakas tlen ne tlapalewilis tlen moitohtok tlanawatilistli weliskiah mochiwas ipan amatlmokuitlawilistli tlen tepostlapolli tlen nochih tlaltipaktli, eliskiah ipatih momanextis tlen ne tlahtlakolli tlen politokeh kietichiwah mokawah kitilanah ika ne itlamilis tlen ome mochitok, axikuentah tlah ne tlakuatopewalli tlen ne moseltiowiyah wahahih sempualli wan nawi kalkoh otlah pilkentzih.

Tlen ne wahapah momanextihih kiistosnekih tlen, kemah tekiwatlanawatihetl wan itlakayowah tlen temiktianih tlakualtlalistli, tlayekanketl ika ne tlen eltoyah weyihtlanawatihetl tlatilanketl, kikixtiliah tlen ne moseltiowiyah tlen ne normalistas ika itlamilis tlen seyoh kitlaliseh tlen ne temanawihetl tlen iwikal topatih, wan axteheliah tehelihetl tlen itlachialis, wahayah ipan nopayah kemah mokatok mochihih ne tlahtlakolli, kampa axikuentah ne tonatih tlen kixikotokeh tlapeyontilistli tlen moseltiowilli.

Kampa kualli mokuamachilis ika namah tlamanextilli monekih mamochiwah tlaixnexamostlahtolli ipan kualli tlasenkawaltlahtolli. Tlah tichiwaseh nikanih ne tlamantlih tlen temanawihetl tlen ne iwikal topatih otlen ne moseltiowiyah nehnehmitekipamitl tlen ne ixnexkayotilli, nohiah, -kampa kiitzkitok Galaín-ne moseltiowilli motlalolli tlen ne masehmelawakayotl, moitah tlen nochih kualli tlen ne tlamantlihtlen temanawihetl mokawah tlawel tlakohkolih kemah, ika itlamilis tlen tlakuatopewas tlen ne temanawihetl tlen kualli tlasenkawaltlahtolli, ne tlakohkolli elih sah tlenyak tlen ne moseltiowilli wan axkinekih tlen panotok, kehnopah kampa sakatiahah tehelihetl ika itlachialli, ika itlachialistli ne tlakuilohemeh. Kampa moixnextiah, ni tlanechikolistli mokestok henih ipan se xiwitl eltok.

\section{III. ¿Yah ne politokeh tlen ne ompualli wan eyi momaxtianimeh tlen Ayotzinapa se tlamantlih tlen tlaixnextilistli tlen echkah ika tlayekanalih tlen ne kinekih ipan tepostlapolimeh tlakualtlalismeh tlen tlanawatihetl?}

Ika ikoyahah moixnextihih ipan ne nechikolistli tlen ne melawaksahetl, ne tlapoalistli tlen ne tlaixnextilistli ika tlayenkawilli tlen ne kinekih ipan tepostlapolimeh tlakualtlalismeh tlen tlanawatiyah, mochitok ika Claus Roxin ipan $1963^{27}$, mokuapkih tlen ne kenihatzah mochiwas temiktikemeh -tlen tlakotontlih iwikal ipan echkah nikanih-, ika wapalitleholtilistli tlen nochih tlaltipaktli. Tlamantlimeh ika kenihatzah tlen ne mikilistli tlatehelwilli a Eichmann ${ }^{28}$ otlen Staschynskij ipan Alemania, polihemeh, tlaixtehemeh wan mikilismeh tlatehelwilli a Alberto

\footnotetext{
${ }^{26}$ Ambos/Böhm; Ne tlaixnextilistli sahetl ...: ob.cit. p.41.

${ }^{27}$ Ipan itlachiwal; Täterschaft und Tatherrschaft, 1a edición, 1963, Walter de Gruyter; pp. 242 y ss. Tlen sehah xiwitl, itlanechikotlahtol tlawel kuehketzih kampa tlawel itlamilli, «Straftaten im Rahmen organisatorischer Machtapparate», Goltdammers Archiv für Strafrecht; 1963, pp. 193 y ss.

${ }^{28}$ Xitlachilikah ne tlakakilistli tlen Roxin tlen iwikal; Straftaten...; ob.cit.p. 193-201. Tlen tlawel kenihatzah tlanehnehchikotlahtolli, motlatowah tlen kikonantok tlen ne itlakayowah tlen Consejo tlen Defensa Nacional ika ne tlatopontlih tlen mochitok tlen ahiahah iniwantih kinekiayah witoniseh ipan ne tlatzaktlih tlen kinxeloyahyah ne ome alemanias.
} 
Fujimori Fujimori ${ }^{29}$, tlen eltoyah weyihtlanawatihetl ipan ne Perú ${ }^{30}$, otlen ne expoalli wan matlaktli wan chiknawi mikilismeh tlatehelwilimeh ipan tlayekanketl tlen Sendero Luminoso ${ }^{31}$ ipan ne sehah weyihaltepetl, sah se tlaixnextilistli tlen ne tlaixnexkayotilli tlen kikualnamikih tlen ne tlapoalistlih Roxiniana kipiah ipan ne Procuración wan Impartición tlen Justicia. Ne tlaixnexkayotilistli tlen ne tlapoalistlih welih moitah, nohiah, ipan tlen ne moitowah tlen, kiseliah ni tlalamikilistli, teheltok ipan se ome xiwitl ne Corte Penal Internacional ipan ne tlamantlih Katanga $^{32}$, kitlahtlakolitokeh ika temiktianimeh tlen kualantli wan ayohkualli tlaltipaktli ${ }^{33}$.

Roxin kimanextiah, kampa tlen ne se tlen tlatzinpewaltilli tlen tlayekanketl tlen itlalamikilis, tlen «la decisión acerca de si se es autor o partícipe, no puede depender de las valoraciones de

29 Se tlen ne tlatehelwilli ipan Ex Presidente kipiah iwikal tlen ne mochitok tlen Ayotzinapa, tlen ika kentzih kiitaskiah; tlen itlalamikilis, xitlachilikah ne tlakohkolmeh tlamanextilimeh ika Caro Coria, Dino Carlos; tlen ne tlatzakuiltilistli tlen eltoyah weyihtlanawatihetl Alberto Fujimori Fujimori ika tlakuilohetl tlen se tlakualtlalistlih temiktihetl nochimeh; ZIS-11/2009; «El mismo destacamento colina fue el autor directo de otro de los casos materia del proceso, llamado caso «La Cantuta». Kehnopah, ne 18.7.1992, ni tlanechikolli kalahih ipan ne Universidad "Enrique Guzmán wan Valle” -ika kiixmatih «la Cantuta»-, moexkawiyah tlen ome teposnehnenketl pick up, mo kualtlalistokeh ipan miyakeh tlanechikolli, nochih iniwantih moixpiktokeh wan kiwikah tepostlatopontlih tlen titl. Yaheh ipan inichanse tlen ne momaxtianimeh, kinkixtiheh kampa kochiayah wan kinostiaheh ika tlawasantli ipan tlatzinpewaltilli, kinkixtihtiaheh chihnawih momaxtianimeh ipan ne tlasentilli. Nopekah tlen ne tekitl, kiskeh wan kiniwikakeh tlen tehewah ipan ne teposnehnenketl tlen motokaxtitoyah, wan kemah moasiyahyah ika Km. 1.5 tlen ne kampa motlaloah teposnehnenketl Ramiro Prialé, kinketzkeh ne teposnehnenketl wan temokeh ne teitzkianimeh. Elkih kampa ne tlanechikolli kampa kinimiktiheh wan kintlalitoheh; p.581.

${ }^{30}$ Ika itlachialis; Pariona Arana, Raúl; Ne iselti tlen echkah ika tlakualtlalistlih ipan ne Sentencia ika Fujimori; www.zis-online.com (11/2009); «El 7.4.2009 la Corte Suprema del Perú condenó al ex presidente Alberto Fujimori Fujimori como autor mediato de crímenes contra la humanidad por el asesinato y posterior desaparición de un profesor y nueva estudiantes de la Universidad Enrique Guzmán y Valle (La Cantuta), por la muerte de diecisiete personas en una quinta del distrito limeño de Barrios Altos y por el secuestro de un empresario y un periodista. Para fundamentar el tipo de participación criminal que desplegó el ex mandatario peruano, la sentencia se basó en la teoría de la autoría mediata por organización»; p. 609. tlamanextilih, ipan itlachialis, Jakobs, Günther; kampa yah kiitzkitok ne tlamaxtilli tlen tlatehelwilli ika coautoría; Zur Täterschaft des Angeklagten Alberto Fujimori Fujimori; ZIS 11/2009; p. 572. Nohiah; Velásquez Velásquez; Fernando; «la teoría de la autoría mediata no se puede sostener porque es sofista y ambigua: da por hecho que existe una única forma de construcción de los aparatos organizados de poder criminales, lo cual no es correcto. Asume que todos los aparatos funcionan de la misma manera a pesar de que está claro que son diferentes y que cada uno tiene sus propias características»; Paramilitärische Führer und kriminelle Machtapparate; ipan; Streitbare Strafrechtswissenschaft; Festschrift für Bernd Schünemann zum 70 Geburtstag; Roland Hefendehl, Tatjana Hörnle und Luis Greco; De Gruyter, Berlin 2014; p. 1130. Tlamanextilih, ika ihatzah sehah tonatih kiseliah ne tlen ipatih tlen ne kiitowah roxiniana; Caro John, José Antonio; Manual teórico-práctico de teoría del delito; Instituto Max Planck para el Derecho Penal Extranjero e Internacional; 2014; p. 206.

${ }^{31}$ Abimael Guzmán; tlayekanketl tlen ne tlakualtlalistlih temiktihetl.

${ }^{32}$ Ipan itlachialis, Ambos, Kai; tlen ne «organización en el dominio de la organización»; InDret 3/2011; p. 12; «En el proceso de la CPI contra los líderes milicianos Katanga y Ngudjolo Chui, se aborda la imputación de crímenes contra la humanidad y crímenes de guerra que fueron cometidos en febrero de 2003 , en un ataque conjunto de ambos grupos milicianos por miembros de las asociaciones lideradas por dichos procesados, en donde la estructura de mando de ambos grupos estaba estrictamente dividida (por la pertenencia étnica disímil de sus miembros). No obstante, la Sala de Asuntos Preliminares de la CPI imputó a los procesados -mediante una curiosa combinación de autoría mediata (dominio de la organización) y coautoría como coautoría mediata ("mittelbare Mittäterschaft) (indirect co-perpetration)- no sólo los hechos de los propios subalternos, sino también los de cada uno de los otros, ya que habían intervenido en virtud de un plan común, siempre habían efectuado una contribución fundamental y habían controlado conjuntamente la totalidad del asalto».

33 Tlamanextilih ipan iyawalis tlen ne tlapoalistlih tlen tlayekanah tlen panotok -ika tlaixnextlahtolli chikawak-; Kindhäuser, Urs; «De esto resulta lo siguiente: si el dominio del hecho se identifica con la capacidad de acción necesaria para evitar la realización del tipo, entonces es claro que el dominio del hecho sólo puede estar referido al propio comportamiento contrario a deber, y nunca a un comportamiento contrario a deber de alguna otra 
cada juzgador en lo individual, sino que dependerá exclusivamente de los presupuestos objetivos de la ley» ${ }^{34}$. Ipan ne tlapoalistlih tlen tlayekanketl tlen panotok, ika tlayekanketl tlen ne kinekih ipan tepostlapolli tlakualtlalistlih tlen tlanawatihetl, motlatoskiah -ika itlachialis Roxin- ne tlamantlih tlen iselti tlen echkah ipan ne tlayekanketl -tlen ahiahah tlayekanah ne tlakualtlalistlih- kiwikah nochih ne nemilistli temiktihetl, moitoskiah, kampa «la organización funciona automáticamente sin que sea necesario que el líder se haga presente» ${ }^{35}$. Kehnopah, eliskiah nawi ne tlapalewiyanih tlen kichiwah ne tlapoalistlih moitohtok: se. Ne tlanawatihetl tlen tlayekanah, ome. Ne tlaiyokalli tlen xitlawaktlahtolli, eyi. Ne tlatekowilli tlen ne temiktianih, wan nawi. Ne monekuilohetl tlen ne tlachiwalistli tlen mochitok chikawak ${ }^{36}$. ¿Ipan tlechketl mopalewiyah ni tlatzinpewaltilli-wan ipan tlah welih mochiwah- kenihatzah mochiwaskiah ipan tlamantlih tlen ne normalistas tlen Ayotzinapa? Ne weyihtlayenkalsahetl masewalli kiitzkiah se tlalamikilis axiwikal tlen ne kiwikah temiktihetl tlen kiitah ika tlakuilowanih wan iwampoyowah. Kehnopah, ne tlaixnextilistli kichiwah se kenihatzah tlen tlaixnextilistli tlayekanketl tlen moixnextis kemah ne tlahtlakolli mochitok ika ne «hombre de atrás» wan «autor de escritorio», ika senikoneh wampoyowah tlen kichiwah moitzkiah.

Notlachialis tlen, ika tlatzinpewaltilli ipan ne tlapoalistlih tlen Roxin, monekiskiah momanextis tlen ne weliskiah tlen tlatehelwilli ne keskih tlen ne tlahtlakolli tlen nikanih moitah- ni motlaliah ipan tlahoh tlen politok kietichiwah tlen wampoyowah-, ika tlatzinpewaltilli ipan ne tlayekanah tlen axkualli tlen temiktihetl oneh tlayekanah ika tlatewihayotl tlen ne kinehih, tlen welis motlahtlahotlalis ne tlahpihpialli ipan ne tlen kinekiayah ipan ne moiliayah tlen tlayekanah tlen ne kinekih. Wan yainih kehnopah, kenkeh, kampa kenihatzah moiyokatlaliah tlen ne tlahtolitlahtlanketl mochitok ika ne Procuraduría General tlen ne República, tlen nehneliah tlakuiloyanih tlen ne politokeh tlen ne normalistas- axkanah sah iniwantih tlen tepalewiheh ipan ne tlen panotok- eliskiah tekiwatlanawatihetl. Moitoskiah, altepetlayekananih, ipan itlanawatil tlen ne Presidente Municipal, tlen ahiahah istoyah ipan ne itlamilli tlen kimaxtitokeh weyihkaltlamaxtiloyah, tlen nochimeh iniwatih eltokeh iniwanpoyowah tlen Estado. Xitlachikiliakah, ne tlanechikotlahtolli 29 tlen ne Ley Orgánica tlen Municipio Libre tlen Estado tlen Guerrero:

Los ayuntamientos, a propuesta de los Presidentes Municipales, nombrarán a los siguientes servidores públicos, sin perjuicio de la denominación o rango jerárquico que los propios Ayuntamientos establezcan:

IV.

persona (...) En ningún caso puedo dominar, a través de mi contribución, una infracción del deber ajena. (...) En la medida en que la teoría del dominio sostiene algo distinto, ella finge hechos y atenta así contra el principio de culpabilidad»; ipan, Infracción de Deber y Autoría-se Crítica tlen ne Teoría tlen Dominio tlen Hecho; Traducción tlen Juan Pablo Mañalich R; Revista tlen Estudios tlen ne Justicia; No 14 - año 2011; pp. 46-47.

${ }^{34}$ Straftaten ...; ob.cit.; p. 197.

${ }^{35}$ Straftaten...; ob.cit; p. 200.

${ }^{36}$ Roxin kichiwah ni nawi tlamanextilih - moitohtok ika kampa itlachiwalli tlen 1963 - ipan itlamanextilih ipan ne moitohtok tlen Fujimori; Bemerkungen zum Fujimori-Urteil des Obersten Gerichtshof in Peru; ZIS 11/2009; p. 565 . 
Jefe de Seguridad Pública, quien deberá reunir los requisitos previstos en la Ley de Seguridad Pública del Estado de Guerrero, además de ser previamente evaluado, capacitado y certificado por las estancias (sic) estatales competentes.

Tlen ne nehneliah tlakuiloyanih maelikah itlakayowah tlen weyihaltepetl axkanah kikixtiah ne weliskiah, ika sah mochitok, mamochiwah tlen ne sekinokeh kenihatzah tlen tlakuilolimehika axkualli oika tlatewihayotl- tlen ne mochitokeh tlaixnexamostlahtolli. Yonseh tlaixnexamostlahtolli onkah tlen kiwikah ne moilistli, ipan ne tlaixmatilkayotl tlen kemah tlatoponistli wan kikixtiliah tlen ne moseltiowiyah tlen ne normalistas, ne tekiwameh tlen tlatilantok tlen Iguala wab Cocula moasitoskiah «coaccionados» exkapah se «error» ika iyawalis ipan tekiwah tlen kichiwayahyah. Ika ne iyawalli, ne tlahtolitlahtlanketl tlen ne Procuraduría General tlen ne República, tlen mochitok nochimeh moitohtok tlen ne tlatehelwilli wan namah monemiliah, mokawilis mamonekih tlen ne tekiwatlanawatihetl kichiwayahyah ika kualli tlaixmatilkayotl tlen kichiwayahyah: timatoyahyah tlen ne normalistas eliayah momaxtianimeh, tlen yuwiyahyah ipan se tlamanextilli tlasentilli tlen teposnehnekemeh wan tlen kiwikayahyah tlaixnexkayotihetl tlen weyih tlanawatihetl, moistoskiah, tlen namah tlatehelwilli wan tlen eliayah weyihtlanawatihetl tlatilanketl tlen Iguala wan isiwah. Yainih kipiliwiltiah sakatiahah moilistli tlen axkualli otlen tlatewihayotl, ika notlachialis, weliskiah mochiwas ne tlaixkompikayotl tlen ne tlaixnextilistli, ika tlayekanah tlen kinekiayah, tlen kualkistok ika ne nawi tlen monekih tlen wahapah momanextitok. Nochih yah, ipan ne kuamachiloyah tlen kemah onkah ne mochitokeh istoyah se tlakualtlalistlih tlen nochimeh tlanawatilli: ne «Municipio Libre de Iguala de la Independencia», itekiwatlanawatihetl wan ne tekiwatlanawatihetl tlen Cocula ${ }^{37}$.

\section{Tlamaxtilimeh tlatehpanihtalli:}

\section{Ne tlanawatihetl tlen tekiwah}

Ne tlanawatihetl tlen tekiwah ne se tlapalewilli tlen ne tlaixnextilistli tlen echkah. Ni moixnextiah ipan ne tlaixnexkayotilistli kampa kipoas tlanawatilli wan mamochiwah. Ika itlahtol tlen Roxin; «basta que el líder de la organización presione un botón, para que el resultado se produzca, sin necesidad de que el líder conozca al autor material. Sin que sea necesario que acuda al error o a la amenaza ${ }^{38}$ ». Ika ihatzah kikalakiah sekih tlaixmatilkayotl axkanah kiitzkitok Roxin, ne moitohtok kipotok ika ne Sala Penal Especial tlen ne Corte Suprema tlen Justicia tlen ne República tlen Perú, ipan ne tlamantlih Fujimori, monextiah ipan kemah kichiwah itlaixnextlahtolli tlen ni axtowih tlatzinpewaltilli:

«... la denominada autoría mediata se presenta cuando el hombre de atrás se aprovecha de los sujetos que se encuentran subordinados a otros en un aparato organizado de poder de tal suerte que por esa vía el primero mantiene un dominio objetivo del hecho -autoría mediata por dominio de la organización-; que ésta última se sustenta en dos elementos esenciales: existencia de un aparato de poder estructurado y la predisposición de los ejecutores; que el acusado tuvo una intervención vertical en los delitos imputados - ejecutados materialmente por efectivos de inteligencia militar en torno al Grupo

\footnotetext{
37 Ome tlatilankemeh, tlen wan motzkitokeh, tlen Estado Mexicano tlen Guerrero.
}

${ }^{38}$ Straftaten; ob.cit.; p. 200. 
Colina y al SIE-, en los que se dio una división de funciones y una línea jerárquica en la organización, en cuya cúspide se encontraba». ${ }^{39}$

Ipan tlamantlih kampa ne tlen Perú ika ne Presidente tlen República o ipan Iguala ika ne weyihtlanawatihetl tlatilanketl, timoasikeh ika se wampoh-ne tlayekantok tlen ne tlakualtlalistlih-, tlen moixnextiah ipan itlamilis tlen ne tlahtoktli, kinexkayotitokeh ika ne tlaktipaktli ika kenihatzah tlakuilohetl tlen tlamaxtilli: ni tlanawatiyah wan tlen nopasekih tlen tepostlapolli nochih kichiwah. Ni moitah, nochih tlen ne weyihtlanawatihetl tlatilanketl -namah kitlatelwiliheh- kipixtoskiah, monekih tlen itokayotlalistli, ne tlaixnexkayotilistli kampa temakas tlanawatilli xitlawak ipan itlamaxtilli tlen tlayekanah tlen tlakualtlalistli (tlatilanketl), kehnopah nohiah welis eltos kualli tlen itlachiwalli, tlen monekih ne tlakualtlalistlih tlen tepostlapolli (nochimeh ne tekichiwameh tlen ne tekiwatlanawatihetl tlen tlatilanketl tlen Iguala eltoyah satlewelih kiniltoyah yah, ika itlanawatil itlamaxtilli nehnehlia tlen weyihtlanawatihetl tlatilanketl).

Tlen namah kitlatelwiliheh kipixtoyah ne tlayekantilli tlen ne tlakualtlalistlih, wan kipiah ne itlamaxtilli tlen tlakuilohetl ika tlayekanalli tlen ne kinekih, kikawah kualli axkanah sah ne tlakualtlalistlih tlen motekowiyah tlen tlatilanketl -ipan ne tlen tlakualtlalistlih mochitok nochih ne tekiwah-, nohiah ne tlasentilli temiktihetl ika ne temiktihemeh tlakualtlalihlih tlen ne yah itlahtolitlahtlanketl kimahatoskiah. Ni yah wan isiwah -nohiah kiitzkitokeh wan kitlatelwiliheh- kipiaskiah chikawak mosansehahtlalwilli ika ne tehelistli tlen guerreros unidos-tehelistli ipan tlen ne tekiwatlanawatihetl temakakeh ne normalistas- tlen ne isiwah tlen eltoyah weyihtlanawatihetl tlatilanketl eliskiah tlayekanketl. Ipan ni tlaixmatilkayotl, xitlachilikah ne tlaixnextlahtolli tlen ne Corte Suprema tlen Perú, kinotzah ne Roxin:

En consecuencia, el hombre de atrás podrá confiar siempre en que su orden o designio criminal se van a cumplir sin necesidad de que tenga que conocer al ejecutor inmediato. (...) Por tanto, no será indispensable que exista una disposición expresa y que esté contenida en un documento, por la que el nivel superior estratégico ordene directamente el cumplimiento de una función específica al ejecutor inmediato $^{40}$.

Tlen ni yehyehtzih tlen tlaixnextilli ne «autor de escritorio» axkanah kiixmatih ne tlen nehnehliah tlakuiloyanih tlen tlahtlakolli ipan axtlen kitlahtlakowah ne tlatehelwilli tlen monekilistli. Nohiah, kampa teheliah ne Roxin kemah monechkawiyah ne nemilistli kichiwah a Eichman; «la cercanía o lejanía del autor mediato con el autor material -que mata directamente a la persona- no tiene absolutamente ninguna relevancia de cara a la responsabilidad del primero» ${ }^{41}$. Ne Corte Suprema tlen Perú yuwih achih nekah wan tlamanextiah.

«Cabe destacar, en este contexto, que el grado de reprochabilidad que ha de recaer sobre el titular del poder de mando será siempre más intenso cuando el origen del mismo parte de un marco de legitimidad formal ${ }^{42}$. En estos casos, pues, corresponderá un mayor grado de desvalor, porque aquél, abusando de su posición de dominio produce una doble afectación al sistema al crear y dirigir una estructura organizacional jerárquica y delincuencial, a la vez que paralela y encubierta. Primero, por haberse alejado del orden legal establecido y que era la fuente del uso legítimo del poder; y luego, porque al ser conocedor del marco jurídico existente diseña y activa dicha estructura criminal, de modo

\footnotetext{
${ }^{39}$ Moitohtok; p. 625 .

${ }^{40}$ Moitohtok; p. 633.

${ }^{41}$ Straftaten; ob.cit.p. 202.

${ }^{42}$ Moitohtok; p. 637.
} 
que resulta menos identificable a las autoridades encargadas de la prevención y control del delito. Por lo tanto, todo aquél que en atención a su jerarquía pone en funcionamiento la maquinaria del aparato de poder organizado, para la comisión del delito, deberá responder siempre como autor mediato» ${ }^{43}$.

Mamotlalih ne tlapoalistlih roxiniana ipan tlamantlih Ayotzinapa axkanah kiitztosnekih, kampa kenihatzah tepotlamilih weliskiah moiliah ika ne kaltlamaxtiloyah masewalli, mokawas moseltiowiyah ne tekihmeh tlen mochitok ika ne nehneliah tlakuilowayanih. Ika ikuapilli, kampa kikawah kualli ne Roxin; "la autoría mediata, por dominio de la voluntad en un aparato organizado de poder, deja incólume la responsabilidad de los autores materiales» ${ }^{44}$. Ika sehah tonatih monekih moitas, ipan pakilistlih ika ne Roxin, tlen ipan ni tlaixnextilistli tlen tlamantlimeh «el delito no sería posible sin contar con las personas que se ubican en la punta del aparato» 45; moitoskiah, tlen tlakuilohetl wan tlakuilohemeh tlen nehneliah kichiwah sehah tekitl wan monekih nochimeh kiitoseh ika sehah.

\section{Ne tlaiyokalistli tlen xitlawaktlahtolli}

Ne tlen eltoyah weyihtlanawatihetl tlatilanketl tlen Iguala kiselkih ne tlanawatihetl tlen kenihatzah nehnehliah, ika tlen kemah kitlamakah ipan ne itenoh tlen kikawiltokeh, itekiwah monekih motepanitas nohiah nehnehliah. Ne tlahtolitlahtlankemeh tlen mochitok ika ne Procuraduría General República kimahatoskiah se axmosansehahtlalkeh tzalantik wan xitlawak tlen tlanawatilli tlasenkawaltlahtolli, kemah tlanawatiyah ne inexkayotilli tlen ne momaxtianih wan iteipayah tlen momakak ipan tehelistli tlen ne guerreros unidos -weliskiah tewikah ika isiwah-, moiyokatlaltoyah tlen tlanawatilli tlasenkawaltlahtolli, wan ika ihatzah ika tlatzinpewaltilli ipan ne tlen kualli kemah teheliah ne tlatilanketl, kitekowiyah ne tlakualtlalistlih tlen kualli tekitis ipan ika tlen ne moitohtokeh tlen nochimeh weyihaltepemeh wan tlen ne tlakualtlalistlih. Xikitakah, ipan itlaneltokilli, ne tlaixnextlahtolli tlen ne Corte Suprema peruana:

«Para Roxin, pues, el apartamiento del Derecho no se refiere únicamente al ordenamiento jurídico interno de cada Estado sino también, y muy particularmente, al Derecho Internacional: Sólo porque todos los pueblos del mundo están vinculados a ciertos valores, tenemos la posibilidad de considerar delictivos y punibles las conductas de órganos superiores del Estado que violen de modo evidente los derechos humanos» ${ }^{46}$.

Ika tlen yankuik motekowilli tlen iyolohtlaixnextilli, Roxin kisehatlaliah ne tlen monekih namah mokuanehnemiliah. Tlah kualli neliah ni axtleh mopatlah tlen nikanih moitzkitok, nikitah ipatih nitehelis ipan tlen, tekowah mokuanehnemiloah monekih ne ixnextilistli tlen ni tlatzinpewaltilli, moixnextiah ipan tlaixnextilli tlen mopatlah ipan ne tlamantlih Ayotzinapa. Ni henih eltok, ne tlakuilohetl tlen moixnexkayotihih, kichihih nehnehliah ne tekitl tlen moixnextiah, kiwikayahyah ne tlatilanketl, kichiwayahyah nemilistli tlen nochimeh wan kiitoyahyah ne amatlasenechikolistli kampa kichiwas ipan ne tlatilanketl ipan itekih. Axkanah moitowah ika ne tlahtolitlahtlankemeh mochitokeh ika ne Procuraduría General tlen ne República wan tlen ne Fiscalía General tlen Estado tlen Guerrero, moiliayah ika yah -teipayah tlen kemah tlen mo-

\footnotetext{
${ }^{43}$ Moitohtok; p. 637.

44 Straftaten; ob.cit.p. 200

45 "Bemerkungen zum Fujimori-Urteil des Obersten Gerichtshofs in Peru; ZIS...; ob.cit. p. 566

${ }^{46}$ Moitohtok; p. 641. 
chitok onkatok ipan Iguala- se ome tlanawatilli tlen kiitzkihih, ika tlen mochitok ipan ne kemah istoyah tlen itlanawatihetl. Ni tlen mochitok wan tlatehelwilli momiakilkih ne tlatehelwilli ika tlen ne politokeh tlen ne ompualli wan eyi normalistas. Xitlachilikah, ipan ni tlaixmatilkayotl, ne tlaixnextlahtolli tlen ne Corte Peruana echkah tlen ne axmosansehotlalilih tlen tlanawatilli tlasenkawaltlahtolli ipan ne tlamantlih Fujimori:

«La desvinculación del ordenamiento jurídico en la criminalidad estatal puede darse de dos maneras: Primero, cuando el nivel superior estratégico del Estado decide apartarse por completo del derecho y crear un sistema normativo totalmente diferente que no es reconocido ni aceptado por el derecho internacional (...) Esta modalidad resulta ser la más grave porque se cubre con una aparente legitimidad. Sin embargo, subrepticiamente intenta crear un sistema normativo alterno al legalmente vigente, aprovechando, justamente, sus formas y estructuras para la comisión de delitos graves».

\section{Ne tlatekowilli tlen ne tlakuiloyanih}

Tlen kualkiskeh ika ne weyihaltepetl tlen namah tlen ne tlapoalistlih tlen ne tlaixnextilli ika ne tlayekanah tlen ne kinekih, welis moitos tlen ne tlatekowilli tlen temiktihetl moitah kemah weliskiah mopatlas ika ne wahapah tlawel weyih tlen iixkankoh ne mochitok tlen ne tlanawatilli temiktihetl. Ni kiistosnekih tlen ne tlaixnexkayotihetl moyawaltok ika ne weyih xiwitl, ipan totlamantlih ne weyihtlanawatihetl tlatilanketl tlen Iguala, axkanah onkah yolseh satlewelli tehelistli, sakatiahah ne tlakuiloyanih weltoskia kimpatlah ika sakatiahah seyoh tlen iixkankoh ne itlamilis tlen ne weyih tlanawatilli. Kehnopah, kampa kiitzkiah Roxin, «el autor mediato puede tener la seguridad en la ejecución del hecho, debido a la fungibilidad de los autores» ${ }^{47}$. Tlen kualli petlanih kiitowah ne Corte Peruana:

«De allí que Roxin, al exponer las características de la fungibilidad, resalte, continuamente, que tal condición garantiza al hombre de atrás la realización del evento criminal y le permite, a su vez, el dominio del hecho. El ejecutor es, pues, simplemente una ruedita cambiable de la máquina del poder, un engranaje sustituible en cualquier momento, pero que ocupará un lugar central en la materialización de los acontecimientos ilícitos» ${ }^{48}$.

Tlen axkanah ika tlatewiyah ne tekuihayotl tlen ne tlamaxtilli roxiniana kampa kiixyakanamikis ne kuanehnemilistli a Fujimiori, García Cavero kiitowah, nohiah, ne tlen monekih tlen axtlatlepantilistli: «la fungibilidad del ejecutor significa que éste no opera como persona individual, sino como engranaje mecánico. Esta fungibilidad tiene, como se dijo, una expresión negativa y otra positiva. La primera significa que si el ejecutor se niega a actuar, el mando superior puede disponer de otro ejecutor igualmente eficaz, mientras que la segunda está referida a la posibilidad de elegir entre varios ejecutores en la organización» ${ }^{49}$. Kampa kiitzkitok ne Roxin:

\footnotetext{
${ }^{47}$ Straftaten...; ob.cit.p. 200.

${ }^{48}$ Moitohtok...; p. 645; echkah tlen ne kualantlih tlen tlayekanketl ne kitokaxtihtohkeh tlen se "especialista" (no fungible) kampa mochiwas ne tlen mochitok temiktihetl; Ambos, Kai; «Sobre la organización en el dominio de la organización»; InDret 3/2011; p. 5.

${ }^{49}$ Ne tlaixnextilistli tlen echkah ika tlen tlayekanah tlen ne kinekih ipan tepostlapolli tlen tlanawatiyah tlakualtlalistlih: Ne tlamantlih tlen Alberto Fujimori Fujimori; ZIS, 11/2009, p. 602.
} 
«Tal organización desarrolla una vida que es independiente de la existencia cambiante de sus miembros. Ella funciona de todos modos, de manera automática, sin importar la persona individual del ejecutante» 50 .

\section{Ne tzalantik monekuilolli tlen ne mochiwah tlen mochitok axtlanawatilli}

Tlen teipayah tlen monekih momanextitok ika Roxin tlen iixkankoh mamochiwah melawak itlapoalistlih ne iwikal tlen mamoitah se «clara disposición interna del sujeto a la realización del hecho delictivo». Moitoskiah, ne tlakuiloyanih monekih istoseh -kualli- ika ne tlapalewilih tlen tlahtlakolli: «la pertenencia a la organización, ya por sí misma, da lugar a una tendencia de adaptación. Se espera que cada uno de los miembros se integre. Esto puede llevar a una cooperación irreflexiva en acciones que jamás se le ocurrirían a una persona que no formara parte de tal organización ${ }^{51}$. Kehnopah, ika ixnextilli, kiitowah ne Corte Peruana:

«En términos concretos, esta categoría alude a una predisposición psicológica del ejecutor a la realización de la orden que implica la comisión del hecho ilícito. Ya no es la fungibilidad del ejecutor lo que asegura el cumplimiento de aquella, sino el internalizado interés y convencimiento de este último en que ello ocurra» ${ }^{52}$.

Itlachialis Ambos -tlen ahiahah motekowitok tlen ni tekitl ipan miyak tlaixnextilistli-, «la pertenencia voluntaria a la organización puede justificar una elevada disposición al hecho por parte del miembro individual, pero el dominio del hecho se fundamenta sólo en el dominio de la organización -comprendido en términos colectivos- y sólo a través de éste se media sobre los miembros ejecutores del hecho» ${ }^{53}$. Ipan ne tlamantlih tlen ne normalistas tlen Ayotzinapa namah politokeh, tiistokeh ipan se tepostlapolli tlen mochiwah ika tekiwahmeh tlen ne tekiwatlanawatihetl tlen Iguala, ika itlanawatil tlen ne namah tlatehelwilli wan ipan itlamaxtilli tlen weyihtlanawatihetl tlatilanketl, kinasitoyah ne tlanawatilli tlen itlamilis itlachilli tlen ne tlakohkolli. Ni politoskiah ika imah tlen tekiwatlanawatihemeh wan iwampoyowah tlen temiktianih tlakualtlalismeh, tlen ahiahah kichiwayahyah -axkanah sah ipan ni tlamantlih, ika tlen kenihatzah tlakualtlalistli-, kampa ika se kenihatzah tepostlapolli tlen tlayekanah ipan tlanawatilli tlen weyihtlanawatihetl tlatilanketl wan isiwah. Kehnopah, ika ixnextilli, sekinokeh kichiwayahyah tekihmeh tlen temiktlih ipan Iguala (cartel de los «guerreros unidos»), sekinokeh tekititoskiah ika kenihatzah «halcones» (tekiwatlanawatihetl) ika kampa teixmatlkawah wan kitlatis ne tekitl tlen temiktlih tlakualtlalihlih tlen ne axtowimeh. Ipan ne mochitokeh, tekiwatlanawatihetl wan temiktianih tlakualtlalihlih kichitoskiah mopatlah se sehah tlakualtlalistlih temiktihetl wan eliskiah sehah tlen ne tlakualtlalihlih: se ome amatlmokuitlawilistli ika se Tlaixmachtilli, sekinoh ika se tepostlatopontlih, wan yuwih sehah itlachialis wan sehah weyihtlanawatihetl tlen ne tlakualtlalistlih.

\footnotetext{
50 Tlayekanah tlen Organización wan Resolución ipan Hecho; ipan, La Teoría del Delito en la Discusión Actual; Tlahtolkuapilih tlen Manuel Abanto Vásquez; Grijley, Lima, 2007, p. 513.

${ }^{51}$ Roxin, Claus; Tlayekanah... ob.cit. p. 531.

52 Moitohtok; p. 649.

${ }^{53}$ Ika ne tlakualtlalistlih...; ob.cit. p. 8. 


\section{Tlaixnextilimeh}

Ika notlachialis, ne tlatzinpewaltilli tlen mopatlah tlen ne tlaixnextilistli tlen echkah, ika tlayekanah tlen ne kinekih ipan se tepostlapolli tlakualtlalistlih tlen tlayekanketl, mochiwaskiah ipan ne tlamantlih tlen ne eltoyah weyihtlanawatihetl tlatilanketl tlen Iguala, Guerrero. Kehnopah, tleh namah monemiliah, ika ne tlateholtilli nehnehlia tlen kitekowiyah ika kenihatzah ne altepetlayekananih tlen kualli kitokaxtitokeh, nichitoskiah tepostlapolli se tlaixnexkayotilistli tlen tlapewaltilli ipan ika tlen ne ompualli wan eyi momaxtianih normalistas tlen politokeh. Ipan itlamaxtilli tlen weyihtlanawatihetl tlatilanketl, wan kipixtok ne tlanawatilistli tlen tekiwatlanawatihetl tlatilanketl, kiyawaltoskiah ne tlanawatilli tlen moitohtok tlen "proceder» ipan iniwantih tlen ne momaxtianih tlen kiitowah tlen miyak kenihatzah, kiawiltihtiah kehnopah tlen iyolohtlaixnextilli tlen tekiwah wan teawiltihtiah ne tlatzinpewaltilimeh tlakualtlalismeh tlen eltoyah ika tlen kipiah tlen kichiwas tlatepanitas wan mochiwas matlatepanitah.

Ne tlen eltoyah weyihtlanawatihetl tlatilanketl weltoskiah kitekowiyah, ika moixnextiah, ne tepostlapolli tlakualtlalistlih tlen tlanawatihetl ika ne tewikalli wan ne itlamilli, ipan itlamaxtilli tlen «autor de escritorio», kikawiltoskiah manemih tlen ika axmelawak ne tepostlapolli tlen tlakualtlaliah, mopatlalihlih ika tlawel tlanawatilli tlen weyihaltepetl wan wampoyowah tlen temiktianih tlakualtlalismeh -tlen ipan itlasentilli kichiwayahyah tlen ika kenihatzah iseltih kichiwah-, moiyokatlaliah tlen ne tlen tlanawatilli tlasenkawaltlahtolli tlen nochimeh wan nochih tlen weyihaltepemeh. Kampa kiahaxilis ni itlachialis, tlen namah kitlatelwiliheh kiilkatoyah ne tlanawatilli tlen moitowah tlen kitzinkotonah wan, tlen kenihatzah moiyokakixtiah tlen ne tlahtolitlahtlanketl tlen mochitok ika ne Procuraduría General tlen ne República, kikonantiah itlachiwalih ika tlayowah tlen sempualli wan chikuaseh wan yawalli tlen sankemayah tlen sempualli wan chikomeh tlen septiembre, kampa mokuamachilkih ne tlen mochitok tlantoyahyah.

Ne tlasasekonawatilli tlen moiliayah mochitok ika ne tlayekanalih tlen ne tlakualtlalistlih, nohiah kitlaltoskiah ne tlapalewilistli tlen iwampoyowah tlen temiktiah tlakualtlalistlih moixnextitokeh ika "cartel de los guerreros unidos», ahiahah tekiwatlanawatihetl ika itlamawatil xitlawak tlen eltoyah weyihtlanawatihetl tlatilanketl, temakatoskiah ne ompualli wan eyi normalistas politokeh. Ne tlahtolitlahtlanketl tlen ne «autor de escritorio» kichiwayahyah ika ne tekiwatlanawatihetl wan itlakualtlalistlih ika tehelistli tlen "guerrero unidos», kipalewitoskiah ipan nochih kemah mochihih tlen ne mochitokeh, ika moseltiowilli tlah kiixmatih kemah otlen axkanah tlen ahiahah kichiheh itekipanolistli, ne tlakuilolimeh kipiaskiah ne monekilistlih tlen axtlatepanitalistli, tlah ne se tekiwatlanawatihetl otlen iwampoyowah tlen temiktihetl tlakualtlalistlih axkanah kichiwah ne tlanawatihetl, mopatlaskiah nimantzih ika seyoh, tlen ahiahah kiwikastiah ne tlayekanketl, ika monekih itzalantik tlakualtlalistli ipan tlen panotok temiktihetl.

Ne tlen politokeh tlen ne ompualli wan eyi normalistas tlen Ayotzinapa, ne mikilistlih tlen sekinokeh eyi, ne sempualli wan makuilli tlakohkolmeh, kehnopah tlen ne tlakohkolli tlen wampoh tlen istoyah ipan ne tlanechikolistli tlen kampa panotoyah, kichiwah se temahmahtih tlakayoawiltilli tlen ne masehmelawakayotl, tlen notlachialis, kipatlah ne tlahtlakolli tlen politokeh kietichiwah tlen wampoyowah ipan tlahtolitlahtlanketl ika mikilistlih weyih, tlakohkolmeh wan tlahtlakolli tlakualtlalismeh, mochitok ika exkapah tlen tlatzinpewaltilli tlen ne tlaixnextilistli tlen echkah, ika tlayekanah tlen ne kinekih, ipan se tepostlapolli tlakualtlalistlih tlen tlayekanah. 